\title{
Volume and surface photoemission from tungsten. I. Calculation of band structure and emission spectra
}

\section{Christensen, N. Egede; Feuerbacher, B.}

\section{Published in:}

Physical Review B

Link to article, DOI:

10.1103/PhysRevB.10.2349

Publication date:

1974

Document Version

Publisher's PDF, also known as Version of record

Link back to DTU Orbit

Citation (APA):

Christensen, N. E., \& Feuerbacher, B. (1974). Volume and surface photoemission from tungsten. I. Calculation of band structure and emission spectra. Physical Review B, 10(6), 2349-2372.

https://doi.org/10.1103/PhysRevB.10.2349

\section{General rights}

Copyright and moral rights for the publications made accessible in the public portal are retained by the authors and/or other copyright owners and it is a condition of accessing publications that users recognise and abide by the legal requirements associated with these rights.

- Users may download and print one copy of any publication from the public portal for the purpose of private study or research.

- You may not further distribute the material or use it for any profit-making activity or commercial gain

- You may freely distribute the URL identifying the publication in the public portal 


\title{
Volume and surface photoemission from tungsten. I. Calculation of band structure and emission spectra
}

\author{
N. Egede Christensen \\ Physics Laboratory I, The Technical University of Denmark, DK-2800 Lyngby, Denmark \\ B. Feuerbacher \\ Surface Physics Division, European Space Research Organization, Noordwijk, Holland
}

(Received 28 January 1974)

\begin{abstract}
The electronic energy-band structure of tungsten has been calculated by means of the relativistic-augmented-plane-wave method. A series of mutually related potentials are constructed by varying the electronic configuration and the amount of Slater exchange included. The best band structure is obtained from an ad hoc potential based on a Dirac-Slater atomic calculation for the ground-state configuration and with full Slater exchange in the atomic as well as in the crystal potential. The selection of this best potential is justified by comparing the calculated band structure to Fermi-surface experiments and to optical-reflectance measurements up to 5 -eV photon energy. The temperature and strain responses in the band structure are estimated from band calculations with four different lattice constants. The band structure was determined in the entire Brillouin zone and is applied to a calculation of photoemission spectra from $\mathrm{W}$ single crystals. The nondirect as well as the direct models for bulk photoemission processes are investigated. The emission from the three low-index surfaces (100), (110), and (111) exhibits strong dependence on direction and acceptance cone. According to the present band model there should essentially be no emission normal to the (110) face for photon energies between 9.4 and $10.6 \mathrm{eV}$. Experimental observation of emission in this gap, however, implies effects not included in the simple bulk models. In particular, effects arising from surface emission have been considered, i.e., emission of those electrons which are excited in a single-step process from initial states near the surface to final states outside the crystal. The electrons that are emitted from the surface in directions perpendicular to the crystal planes carry information on the one-dimensional surface density of states. The present work includes a crude estimate of this surface density of states, which is derived from the bulk band structure by narrowing the $d$ bands according to an effective number of neighbors per surface atom. Estimates of surface relaxation effects are also included.
\end{abstract}

\section{INTRODUCTION}

The present paper describes a calculation of the energy-band structure for tungsten and its application to photoemission experiments. The calculation was performed by an augmented-planewave (APW) method along the general lines laid out by previous work on transition metals. ${ }^{1-8}$ In order to permit the computation of optical and photoemission spectra the band calculation was carried out over the entire Brillouin zone. It was imperative to include relativistic effects in the present calculation. This is obvious from the work of Loucks, ${ }^{4}$ who demonstrated that the calculated Fermi surface of tungsten varies considerably as relativistic effects are taken into account.

The choice of the method for constructing the crystal potential still represents the major difficulty in $a b$ initio calculations of the band structure of the transition metals. Although exchange and correlation effects have been studied extensively theoretically, the results do not suggest any unique way of determining a one-electron potential for band calculations. Further, the theoretical foundations of a particular choice of potential in a cal-

culation like the one presented here is questionable due to the fact that it is not carried through to self-consistency. Also, the electronic configuration in the atoms that form the crystal lattice cannot be chosen unambiguously. ${ }^{2,9}$ In the present approach therefore a number of ad hoc muffin-tin potentials have been constructed from superimposed atomic potentials and charge densities in a manner described earlier, ${ }^{8-12}$ including a $\rho(\vec{r})^{1 / 3}$ term, $\rho(\vec{r})$ being the local electron density. These potentials vary in terms of the electronic configuration and the Slater exchange term. A "best" potential is chosen from this set by means of a comparison to experimental results such as Fermisurface measurements, ${ }^{13-16}$ optical absorption, ${ }^{17}$ and photoelectric emission spectra.

A second set of potentials has been constructed where the lattice constant was varied such that they refer to different temperatures of the crystal. This is desirable for two reasons. First, Fermisurface measurements are made at low temperatures, while optical and photoelectron results are reported for ambient temperature. A low-temperature calculation is required therefore to account for temperature effects on Fermi-surface topology. Second, calculations for different lattice con- 
stants allow an estimate of the strain response of the material. Stress modulation experiments have been shown to yield information on critical point transitions in specific regions of $\vec{k}$ space. Such experiments allow a much more direct comparison $^{12}$ to band-structure calculations than unmodulated optical spectra, which are related to quantities integrated over the entire $\vec{k}$ space (joint density of states). In the hope that future modulation experiments will be carried out, estimates of the strain response parameters for several interband transitions have been included here.

Photoemission experiments usually yield results related to optical excitations over extended regions of $\overrightarrow{\mathrm{k}}$ space, and the interpretation requires a knowledge of the detailed band structure even if extremely simplified models for the photoemission processes are used. It is expected that photoemission experiments confined to a narrow emission angle reduce the extent of $\vec{k}$ regions involved, thus facilitating direct comparison with the band structure. However, such an analysis requires, a priori, a method for deciding which features of the spectra can be attributed to bulk effects and thus be related to the bulk band structure. Many photoemission properties of the noble metals ${ }^{11,18-22}$ have been interpreted to date in terms of direct optical transitions between band states followed by transport of the hot electron to the surface and subsequent emission. The success of this simple model which reflects only bulk properties seems to be limited, and in particular it is not found valid in the case of rhodium. ${ }^{8}$ Recent experiments ${ }^{23,24}$ have shown that the photoelectron energy distribution for emission confined to a narrow solid angle and measured normal to three single-crystal faces of tungsten may be interpreted in terms of both bulk and surface $e^{25-27}$ contributions to the observed spectra. The band structure presented here will be applied in the analysis of those experimental features related to the bulk contribution. ${ }^{23}$ Emission from the surface reflects the density of initial states confined to the surface region. This function, the surface density of states, is related but not identical to the bulk density of states. A simple model giving the surface density of states by scaling the width of the bulk $d$ bands according to the number of nearest and next-nearest neighbors to a surface atom will be presented.

The paper is divided into three main parts. Section II is a presentation of the calculated band structure and the influence of configuration changes and the response to hydrostatic lattice deformations. The actual choice of a "best" crystal potential is justified in Sec. III by means of Fermi-surface calculations and a derived $\epsilon_{2}(\omega)$ spectrum. The connection to the experimental work of Ref. 23 follows in Sec. IV, where the photoemission calculations are discussed. The angular variation of the direct model energy distribution curves (EDC's) and bulk nondirect EDC's as well as the full-angle results will be shown. Further, the crude model for obtaining the surface density of states is included in Sec. IV.

\section{ENERGY BANDS OF TUNGSTEN}

The electronic energy-band parameters of tungsten have been examined for eight different crystal potentials. The characteristic parameters of these potentials are given in Sec. II A. Four of the potentials $\left(V_{1}, V_{2}, V_{3}, V_{4}\right)$ are constructed in the same way except for varying the lattice parameter. One of these potentials $\left(V_{2}\right)$ yielded a band structure that is described in some detail (Sec. II B) since it shows the best agreement with experimental data. This particular band structure, together with the results obtained using $V_{1}, V_{3}$, and $V_{4}$ gives information on the temperature and strain responses in the energy bands of tungsten (Sec. IIC). The four remaining potentials, $V_{5}, V_{6}, V_{7}$, and $V_{8}$ all refer to room temperature, as does $V_{2}$, but they differ with respect to the amount of exchange which is included and with respect to the electronic configuration of the isolated atom. The band parameters, in particular those concerning the $d$ bands, are discussed (Sec. IID). The band structures corresponding to the potentials $V_{5}, V_{6}$, $V_{7}$, and $V_{8}$ have only been calculated along a few symmetry lines, and the band parameters are essentially described in terms of the logarithmic derivatives of the partial waves for each value of the azimuthal quantum number.

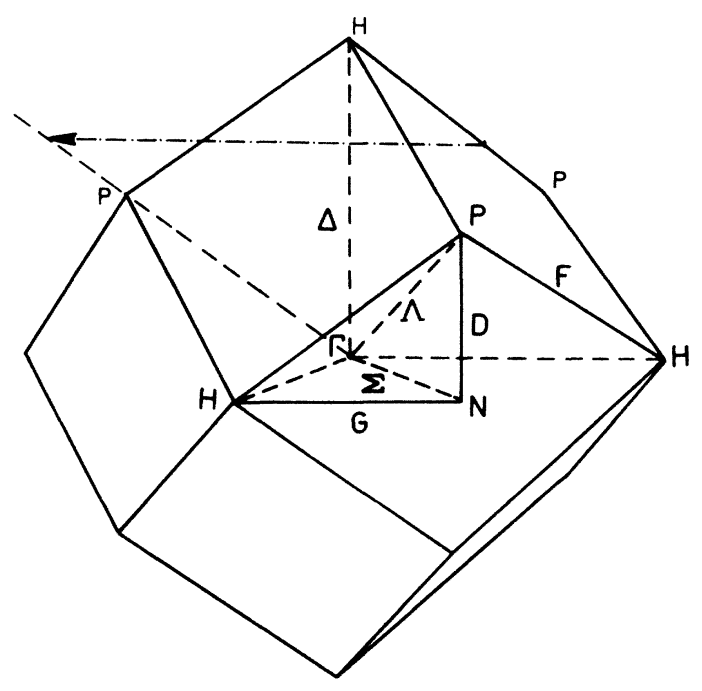

FIG. 1. First Brillouin zone for a bcc lattice. The line marked (dot-dashed line) shows a $(1,-1,0)$ reciprocal-lattice vector that connects an $F$ point to a point on the continuation of a $\Gamma P$ line (cf. Sec. IV). 


\section{A. Crystal parameters and potential}

Tungsten crystallizes in a bcc lattice, and Fig. 1 shows the first Brillouin zone with the irreducible $\frac{1}{48}$ zone to which the calculation can be restricted. The calculated band structure will be compared to room-temperature optical measurements and to Fermi-surface experiments which are carried out at low temperatures. Ther efore, band calculations are required for $T=300 \mathrm{~K}$ as well as for $T=4.2 \mathrm{~K}$. The room-temperature lattice constant is ${ }^{28} 3.1651 \AA$ (5.9811 a. u. ), and, using the thermal expansion data of Nix and Mac Nair, ${ }^{29}$ the 4. 2-K lattice constant has been estimated ${ }^{5}$ to be 3. $161 \AA$ (5.973 a. u.). For the purpose of examining strain responses and estimating surface-relaxation effects, we have performed band calculations for two expanded crystals corresponding to the lattice parameter $a=6.100 \mathrm{a}$. u. and $a=6.400 \mathrm{a}$. u.

The crystal potential has been constructed in a manner similar to the one used for $\mathrm{Au}^{12}, \mathrm{Ag}^{10,11}$, $\mathrm{Cu},{ }^{30} \mathrm{Mo}^{9}, \mathrm{~V}^{9}, \mathrm{Rh},{ }^{8,31}$ and $\mathrm{Pd},{ }^{31}$ starting from relativistic Hartree-Fock-Slater calculations of the atom. The atomic charge densities were applied to construct an atomic Coulomb potential by solving the Poisson equation, and the Coulomb contribution to the muffin-tin potential was obtained by superposing and averaging ${ }^{32}$ atomic Coulomb potentials from 14 shells of neighbors. The exchange term has been derived from the cube root of a muffin-tin charge density calculated by superposing atomic densities inside the muffin-tin spheres and smearing out the remaining charge ${ }^{10}$ between the spheres. Thus the Coulomb and exchange contributions were treated separately. The constant potential between the muffin-tin spheres consists of an average Coulomb potential plus the exchange potential calculated from the average electron density. The averaging of the Coulomb potential was not completely strict, but rather a simple Wigner-Seitz sphere averaging was used.
The radius $R_{s}$ of the muffin-tin spheres has been chosen to be 2.459 a. u. for all potentials, implying that even for the lattice with the smallest nearest-neighbor distance the spheres are not touching each other.

Table I gives a list of characteristic parameters for the potentials that have been used in the present work. The constant $\alpha$ is the weight of the $\rho^{1 / 3}$ exchange term such that $\alpha=1$ corresponds to full Slater exchange. $V_{0}$ is the constant potential between the muffin-tin spheres and $\Delta V_{0}$ gives the discontinuity at $r=R_{s}$. The lattice constants $a$, the atomic volumes $\Omega$, and the Wigner-Seitz sphere radii $R_{\mathrm{WS}}$ are also included in the table $\left(\Omega=\frac{4}{3} \pi R_{\text {ws }}^{3}\right)$. Two electronic configurations in the atom have been considered, $5 d^{4} 6 s^{2}$ and $5 d^{5} 6 s^{1}$. The former corresponds to the ground-state configuration of the isolated tungsten atom.

B. Band structure calculated from $V_{2}$

The diagram in Fig. 2 shows the band structure of $\mathrm{W}$ along symmetry lines in the Brillouin $\mathrm{z}$ one (Fig. 1). The energy eigenvalues have been obtained by the relativistic-augmented-plane-wave (RAPW) method in the same way as described earlier. ${ }^{12}$ At least nine bands were calculated at 285 $k$ points uniformly distributed throughout the irreducible $\frac{1}{48}$ zone (Fig. 1). Along symmetry lines additional points were included such that the density was doubled. The eigenvalues at 55 points uniformly distributed throughout the $\frac{1}{48}$ th zone are listed in Table $I$. The band structure of Fig. 2 will be the one discussed in most detail since it gave the best agreement with experiments. The potential $\left(V_{2}\right)$ corresponds to the ground-state configuration of the atom and full Slater exchange $(\alpha=1)$ was included in the atomic as well as in the crystal potential (Table I). Although this calculation is for the room-temperature lattice constant it is possible to draw some conclusions concerning the qualitative features of the Fermi surface. The

TABLE I. Characteristic parameters for the potentials that have been examined. $V_{0}$ is the muffin-tin zero, and $\Delta V_{0}$ gives the discontinuity at the muffin-tin sphere $\left(r=R_{S}\right) . \quad R_{W S}$ is the Wigner-Seitz radius and $a$ is the lattice parameter.

\begin{tabular}{|c|c|c|c|c|c|c|c|c|}
\hline Label & $V_{1}$ & $V_{2}$ & $V_{3}$ & $V_{4}$ & $V_{5}$ & $V_{6}$ & $V_{7}$ & $V_{8}$ \\
\hline Configuration & $5 d^{4} 6 s^{2}$ & $5 d^{4} 6 s^{2}$ & $5 d^{4} 6 s^{2}$ & $5 d^{4} 6 s^{2}$ & $5 d^{4} 6 s^{2}$ & $5 d^{5} 6 s^{1}$ & $5 d^{5} 6 s^{1}$ & $5 d^{5} 6 s^{1}$ \\
\hline$\alpha($ atom $)$ & 1 & 1 & 1 & 1 & $\frac{2}{3}$ & 1 & $\frac{2}{3}$ & 1 \\
\hline$\alpha$ (crystal) & 1 & 1 & 1 & 1 & $\frac{2}{3}$ & 1 & $\frac{2}{3}$ & $\frac{2}{3}$ \\
\hline$a$ & 5.973 & 5.981 & 6.100 & 6.400 & 5.981 & 5.981 & 5.981 & 5.981 a.u. \\
\hline$\Omega$ & 106.548 & 106.977 & 113.491 & 131.072 & 106.977 & 106.977 & 106.977 & 106.977 a.u. \\
\hline$R_{\mathrm{WS}}$ & 2.9409 & 2.9449 & 3.0035 & 3.1512 & 2.9449 & 2.9449 & 2.9449 & 2.9449 a. u. \\
\hline$V_{0}$ & -1.4155 & -1.4107 & -1.3427 & -1.1974 & & -1.3905 & & $-1.0689 \mathrm{Ry}$ \\
\hline$\Delta V_{0}$ & 0.1367 & & 0.1430 & & & 0.1173 & & $0.1017 \mathrm{Ry}$ \\
\hline$T$ & 4.2 & 300 & $\sim 2150$ & & 300 & 300 & 300 & $300 \mathrm{~K}$ \\
\hline$\Delta a / a_{300}{ }^{2}$ & -0.13 & 0 & 1.95 & 6.88 & 0 & 0 & 0 & $0 \%$ \\
\hline
\end{tabular}

${ }^{2} \Delta a=a-a_{300}, a_{300}$ being the $300-\mathrm{K}$ lattice constant. 


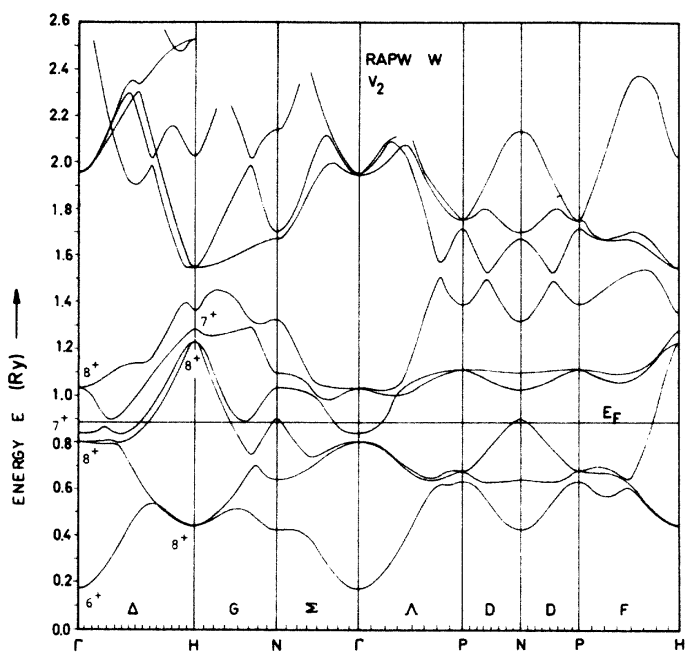

FIG. 2. Band structure of tungsten along symmetry lines as calculated from the room-temperature potential $V_{2}$ which includes full slater exchange. In the atomic calculation the Slater exchange is included with full weight. The electron configuration in the atom corresponds to that of free $\mathrm{W}$ atoms, i. e., $5 d^{4} 6 s^{2}$. The energies are in Rydbergs and measured from the muffin-tin zero $V_{0}$.

Fermi level $E_{F}$ is close to the center of the $d$ bands as would be expected since the $d$ states should be approximately half-filled. Since $E_{F}$ falls in the spin-orbit gap between band ${ }^{33} 4$ and 5 at the $\Delta$ line it follows that no electron lenses exist inside the electron jack. Further, the hole octahedron-still considering the $\Gamma H$ line- is separated from the jack by an amount that compares well to experiments. Also the hole ellipsoids around $N$ exist. Thus, from the diagram of Fig. 2 it is seen that at least the qualitative features of the Fermi surface agree with experiment. ${ }^{13-16}$

The spin-orbit (SO) splittings at $\Gamma$ and $H\left(\Gamma_{25}^{\prime}\right.$ $\left.\rightarrow \Gamma_{7}^{+}, \Gamma_{8}^{+}, H_{25}^{\prime} \rightarrow H_{7}^{+}, H_{8}^{+}\right)$are 0.041 and $0.051 \mathrm{Ry}$, respectively. In terms of a spin-orbit parameter $\xi_{d}$ these energy levels are

$$
\begin{aligned}
& E\left(7^{+}\right)=E\left(25^{\prime}\right)+\xi_{d}, \\
& E\left(8^{+}\right)=E\left(25^{\prime}\right)-\frac{1}{2} \xi_{d},
\end{aligned}
$$

and the splitting is $\frac{3}{2} \xi_{d}$ at $\Gamma$ and $H$. Therefore, the effective SO parameter is different at the two levels $\Gamma_{25}^{\prime}$ and $H_{25}^{\prime}$. In view of earlier calculations ${ }^{18}$ it is not surprising that $\xi$ varies with energy. In the present case we find an effective SO parameter varying from $0.027 \mathrm{Ry}$ at the energy $E=0.816 \mathrm{Ry}$ to 0.034 Ry at $E=1.242$ Ry, $E$ being measured from the muffin-tin zero. Mattheiss ${ }^{5}$ used the experimental value for the splitting between the jack and the octahedron obtained by Walsh and Grimes ${ }^{34}$ to deduce a SO parameter and found $\xi \simeq 0.03 \mathrm{Ry}$, a value between the two numbers given above. The atomic $5 d$ SO parameter is $0.027 \mathrm{Ry},{ }^{35}$ and thus very close to the band-structure values. The difference between the two lowest $H_{8}^{+}$levels is 0.791 Ry indicating that the present calculation yields $d$ bands that are wider than those of Ref. 5 by more than $20 \mathrm{mRy}$. In this comparison we refer to the results obtained using the potential $V_{1}$ of Ref. 5, i.e., where full Slater exchange was included as in the present work. The difference is probably due to the fact that our atomic calculation is relativistic.

At $\Gamma$ we find an almost triply degenerate level at $1.95 \mathrm{Ry}$. It might be argued that this accidental degeneracy between a doubly degenerate level and a nondegenerate level is not significant since the band calculation cannot be trusted too much in this energy region. A free electron with an energy of 2 Ry would have a wavelength of $2 \pi / \sqrt{2}$ a. $u$. $=4.45 \mathrm{a}$. u. which is smaller than the lattice parameter. Therefore, errors in the band calculation due to the muffin-tin shape could be expected in this energy range. In order to examine whether or not this is the case we have calculated the energy bands along symmetry lines using a potential which is identical to $V_{2}$ inside the muffin-tin spheres but with $V_{0}$ reduced by 0.050 Ry. The results at $\Gamma, H$, and $N$ are shown in Table III. The last three columns contain the shift of the energy levels relative to the bottom of the $s$ band at $\Gamma$. Only small shifts are found in the high-energy region, while appreciable effects are seen in the energy range near the Fermi level. Thus, states at energies even higher than $1 \mathrm{Ry}$ above $E_{F}$ are only slightly affected. The shifts near $E_{F}$ can easily be understood. This is the $d$-band range. A shift in $V_{0}$ will produce a change in the potential bar$\operatorname{rier}^{36,12}$ formed by the centrifugal term in the effective potential. Consequently, the tunneling probability will be different and the width as well as the position of the $d$ bands change. In the present case where $V_{0}$ is reduced we have reduced the potential barrier, and this leads to a broadening of the $d$ bands.

The density-of-states function

$$
N\left(E^{0}\right)=\frac{2 \Omega}{(2 \pi)^{3}} \int \frac{d S_{E^{0}}}{\left|\vec{\nabla}_{\overrightarrow{\mathbf{k}}} E(\overrightarrow{\mathrm{k}})\right|},
$$

where the integral is taken over the surface of constant energy $E^{0}$, has been derived from the band structure discussed above and is shown in Fig. 3. It was obtained using the Gilat-Raubenheimer $r^{37}$ method in conjunction with a three-dimensional analog to second-order Lagrange interpolation. ${ }^{12}$ The original $285 \overrightarrow{\mathrm{k}}$ points in $\frac{1}{48}$ zone were interpolated to 3542 points which served as centers for the microcubes. The Fermi level $E_{F}$ $=0.889$ Ry over the muffin-tin zero, was obtained 
TABLE II. Calculated eigenvalues at 55 points uniformly distributed over $\frac{1}{48}$ of the Brillouin zone. Potential $V_{2}$. The energies are in Ry measured from $V_{0}$. (A table of the RAPW eigenvalues at $285 \overrightarrow{\mathrm{k}}$ points may be obtained from the authors.)

\begin{tabular}{|c|c|c|c|c|c|c|c|c|c|}
\hline $\overrightarrow{\mathrm{k}} /(\pi / 4 a)$ & Band 1 & Band 2 & Band 3 & Band 4 & Band 5 & Band 6 & Band 7 & Band 8 & Band 9 \\
\hline$\Gamma(0,0,0)$ & 0.1731 & 0.8030 & 0.8030 & 0.8444 & 1.0350 & 1.0350 & 1.9495 & 1.9505 & 1.9516 \\
\hline$\Delta(0,1,0)$ & 0.2000 & 0.7977 & 0.8070 & 0.8463 & 0.9952 & 1.0488 & 1.9938 & 1.9965 & 1.9987 \\
\hline$\Delta(0,2,0)$ & 0.2751 & 0.7930 & 0.8108 & 0.8642 & 0.9055 & 1.0853 & 2.0934 & 2.1214 & 2.1261 \\
\hline$\Delta(0,3,0)$ & 0.3814 & 0.7671 & 0.8017 & 0.8377 & 0.9274 & 1.1237 & 1.9854 & 2.2043 & 2.2756 \\
\hline$\Delta(0,4,0)$ & 0.4866 & 0.6616 & 0.8347 & 0.8739 & 0.9989 & 1.1352 & 1.9078 & 2.2354 & 2.2986 \\
\hline$\Delta(0,5,0)$ & 0.5376 & 0.5696 & 0.8987 & 0.9454 & 1.0824 & 1.1582 & 1.9727 & 2.0269 & 2.1270 \\
\hline$\Delta(0,6,0)$ & 0.5006 & 0.5119 & 0.9970 & 1.0493 & 1.1654 & 1.2589 & 1.7757 & 1.8889 & 2.1277 \\
\hline$\Delta(0,7,0)$ & 0.4582 & 0.4643 & 1.1256 & 1.1715 & 1.2343 & 1.3886 & 1.5820 & 1.6730 & 2.1174 \\
\hline$H(0,8,0)$ & 0.4440 & 0.4440 & 1.2350 & 1.2350 & 1.2860 & 1.3698 & 1.5500 & 1.5500 & 2.0296 \\
\hline$\Sigma(1,1,0)$ & 0.2254 & 0.7793 & 0.7968 & 0.8734 & 0.9980 & 1.0371 & 1. 9873 & 2.0415 & 2.0630 \\
\hline$(1,2,0)$ & 0.2957 & 0.7598 & 0.7743 & 0.9210 & 0.9454 & 1.0721 & 2.0232 & 2.1223 & 2.1647 \\
\hline$(1,3,0)$ & 0.3932 & 0.7329 & 0.7611 & 0.8904 & 0.9938 & 1.1164 & 1.9335 & 2.1473 & 2.3153 \\
\hline$(1,4,0)$ & 0.4882 & 0.6608 & 0.7893 & 0.8934 & 1.0702 & 1.1446 & 1.8600 & 2.1660 & 2.2754 \\
\hline$(1,5,0)$ & 0.5366 & 0.5877 & 0.8461 & 0.9148 & 1.1515 & 1.2102 & 1.8466 & 2. 0447 & 2.1445 \\
\hline$(1,6,0)$ & 0.5093 & 0.5333 & 0.9286 & 0.9820 & 1.2283 & 1.3412 & 1.7012 & 1.8565 & 061 \\
\hline$G(1,7,0)$ & 0.4734 & 0.4833 & 1.0394 & 1.0937 & 1.2601 & 1.4538 & 1.5645 & 1.6965 & 147 \\
\hline$\Sigma(2,2,0)$ & 0.3492 & 0.7171 & 0.7472 & 0.9924 & 1.0088 & 1.0582 & 1.9462 & 2.0686 & 2.2790 \\
\hline$(2,3,0)$ & 0.4138 & 0.6982 & 0.7381 & 0.9788 & 1.0812 & 1.1147 & 1.8468 & 1.9879 & 2.3972 \\
\hline$(2,4,0)$ & 0.4698 & 0.7070 & 0.7190 & 0.9571 & 1.1421 & 1.1972 & 1.7684 & 1.9673 & 2.2351 \\
\hline$(2,5,0)$ & 0.5077 & 0.6635 & 0.7595 & 0.9061 & 1.2461 & 1.2861 & 1.6985 & 2.0086 & 2.0658 \\
\hline$(2,6,0)$ & 0.5178 & 0.5957 & 0.8345 & 0.9049 & 1.2813 & 1.4046 & 1.6080 & 1.9047 & 2.1688 \\
\hline$\Sigma(3,3,0)$ & 0.4263 & 0.6628 & 0.7970 & 1.0304 & 1.0840 & 1.2 & 1.7694 & 1.8218 & 2.3221 \\
\hline$(3,4,0)$ & 0.4411 & 0.6603 & 0.8238 & 1.0121 & 1.1 & 1.2 & 1. & 1.7816 & 2.1758 \\
\hline$G(3,5,0)$ & 0.4697 & 0.6934 & 0.7925 & 0.9456 & 1.2 & 1.3 & 1. & 1.8708 & 2.0759 \\
\hline$N(4,4,0)$ & 0.4292 & 0.6425 & 0.9015 & 1.0330 & 1.1 & 1.3 & 1. & 1.7019 & 2.1464 \\
\hline$\Lambda(1,1,1)$ & 0.2500 & 0.7670 & 0.7732 & 0.9 & 1. & 1.0 & 2.0007 & 2.0736 & 2.0871 \\
\hline$(1,2,1)$ & 0.3473 & 0.7500 & 0.7500 & 0.9579 & 0.9 & 1.0 & 2.0484 & 2.0639 & 2.1691 \\
\hline$(1,3,1)$ & 0.4086 & 0.7041 & 0.7465 & 0.9167 & 1.0 & 1.1 & 1.9127 & 2.1161 & 2.2760 \\
\hline$(1,4,1)$ & 0.4977 & 0.6479 & 0.7717 & 0.9117 & 1.0 & 1.1 & 1.8249 & 2.1194 & 2.2602 \\
\hline$(1,5,1)$ & 0.5550 & 0.5850 & 0.7878 & 0.9573 & 7 & 1.2 & 1.7786 & 2.0026 & 2.1814 \\
\hline$(1,6,1)$ & 0.5297 & 0.5438 & 0.8328 & 1.0469 & 1.1708 & 1.4383 & 1.6628 & 1.8127 & 2.2647 \\
\hline$F(1,7,1)$ & 0.4931 & 0.4974 & 0.9359 & 1.1670 & 1.1670 & 1.5200 & 1.6101 & 1.6413 & 2.3153 \\
\hline$(2,2,1)$ & 0.3729 & 0.7043 & 0.7198 & 1.0015 & 1.0430 & 1.0770 & 1.9743 & 2.0628 & 2.1882 \\
\hline$(2,3,1)$ & 0.4378 & 0.6808 & 0.7195 & 0.9858 & 1.0885 & 1.1634 & 1.8511 & 1. 9871 & 2.2780 \\
\hline$(2,4,1)$ & 0.4921 & 0.6651 & 0.7355 & 0.9696 & 1.1129 & 1.2707 & 1.7000 & 1.9649 & 2.1792 \\
\hline$(2,5,1)$ & 0.5330 & 0.6479 & 0.7278 & 0.9592 & 1.1582 & 1. 3978 & 1.6580 & 1.9346 & 2.1360 \\
\hline$(2,6,1)$ & 0.5447 & 0.6010 & 0.7449 & 0.9984 & 1.1894 & 1.5170 & 1.5802 & 1.8329 & 2.2410 \\
\hline$(3,3,1)$ & 0.4542 & 0.6556 & 0.7649 & 1.0340 & 1.0879 & 1.2625 & 1.7709 & 1.8229 & 2.1970 \\
\hline$(3,4,1)$ & 0.4674 & 0.6519 & 0.8018 & 1.0220 & 1.1174 & 1.3453 & 1.6662 & 1.7991 & 2.1175 \\
\hline$(3,5,1)$ & 0.4943 & 0.6735 & 0.7530 & 0.9776 & 1.1711 & 1.4082 & 1.6131 & 1.8594 & 2.0793 \\
\hline$D(4,4,1)$ & 0.4565 & 0.6381 & 0.8643 & 1.0426 & 1.1042 & 1.3720 & 1.6362 & 1.7319 & 2.0943 \\
\hline$\Lambda(2,2,2)$ & 0.4332 & 0.6759 & 0.6849 & 1.0276 & 1.0581 & 1.1680 & 1.9797 & 2.0475 & 2.1284 \\
\hline$(2,3,2)$ & 0.4947 & 0.6564 & 0.6842 & 1.0216 & 1.0904 & 1.2706 & 1.8209 & 1.9757 & 2.0557 \\
\hline$(2,4,2)$ & 0.5328 & 0.6412 & 0.7204 & 1.0109 & 1.1037 & 1.3796 & 1.6708 & 1.9199 & 2.0704 \\
\hline$(2,5,2)$ & 0.5759 & 0.6280 & 0.7014 & 1.0259 & 1.1093 & 1.5092 & 1.5828 & 1.8308 & 2.1842 \\
\hline$F(2,6,2)$ & 0.6059 & 0.6205 & 0.6531 & 1.0732 & 1.1034 & 1.5308 & 1.6721 & 1.7060 & 2.3588 \\
\hline$(3,3,2)$ & 0.5302 & 0.6432 & 0.7005 & 1.0546 & 1.0966 & 1.3769 & 1.7099 & 1.7629 & 1.9946 \\
\hline$(3,4,2)$ & 0.5350 & 0.6417 & 0.7486 & 1.0518 & 1.1088 & 1.4615 & 1.5834 & 1.8254 & 1.9913 \\
\hline$(3,5,2)$ & 0.5510 & 0.6517 & 0.7257 & 1.0389 & 1.1175 & 1.5193 & 1.5526 & 1.7906 & 2.0831 \\
\hline$D(4,4,2)$ & 0.5296 & 0.6329 & 0.7904 & 1.0674 & 1.1103 & 1.4820 & 1.5454 & 1.7921 & 1.9557 \\
\hline$\Lambda(3,3,3)$ & 0.6147 & 0.6443 & 0.6521 & 1.0651 & 1.0795 & 1.4869 & 1.6007 & 1.8422 & 1.8865 \\
\hline$(3,4,3)$ & 0.5938 & 0.6557 & 0.7060 & 1.0830 & 1.1127 & 1.4815 & 1.5822 & 1.7838 & 1.8996 \\
\hline$F(3,5,3)$ & 0.5789 & 0.6763 & 0.6992 & 1.0753 & 1.1088 & 1.4638 & 1.6707 & 1.6795 & 2.0115 \\
\hline$D(4,4,3)$ & 0.6126 & 0.6445 & 0.7221 & 1.0957 & 1.1166 & 1.4393 & 1.6207 & 1.7886 & 1.8322 \\
\hline$P(4,4,4)$ & 0.6390 & 0.6810 & 0.6810 & 1.1170 & 1.1170 & 1.3938 & 1.7172 & 1.7578 & 1.7578 \\
\hline
\end{tabular}

by integration of $N(E)$,

$\int_{E_{b}}^{E_{F}} N(E) d E=6$ electrons/atom where $E_{b}$ is the bottom of the $s$ band. The calculated value of the density of states at $E_{F}$ is $N\left(E_{F}\right)$ $=5.04$ electrons atom ${ }^{-1} \mathrm{Ry}^{-1}$. The corresponding 
TABLE III. Relative shifts $E_{2}-E_{1}$ in the band structure due to a shift in the muffin-tin discontinuity. The columns $E_{1}$ give selected energy levels relative to the bottom of the $s$ band at $\Gamma$, whereas the values $E_{2}$ were obtained from an RAPW calculation using the same potential $\left(V_{2}\right)$ except for a reduction of $\Delta V_{0}$ by $0.050 \mathrm{Ry}$. (The levels are numbered with increasing energy.)

\begin{tabular}{|c|c|c|c|c|c|c|c|c|c|}
\hline \multirow{2}{*}{$\begin{array}{c}\text { Band } \\
\text { number }\end{array}$} & \multicolumn{3}{|c|}{$\begin{array}{c}E_{1} \\
\text { Potential } V_{2}\end{array}$} & \multicolumn{3}{|c|}{$\begin{array}{c}E_{2} \\
V_{2} \text { with muffin-tin } \\
\text { zero shifted by } \\
-0.050 \mathrm{Ry}\end{array}$} & \multicolumn{3}{|c|}{$E_{2}-E_{1}$} \\
\hline & $\Gamma$ & $H$ & $N$ & $\Gamma$ & $H$ & $N$ & $\Gamma$ & $H$ & $N$ \\
\hline 1 & 0.000 & 0.270 & 0.255 & 0.000 & 0.280 & 0.264 & 0.000 & 0.010 & 0.009 \\
\hline 2 & 0.630 & 0.270 & 0.469 & 0.648 & 0.280 & 0.485 & 0.018 & 0.010 & 0.016 \\
\hline 3 & 0.630 & 1.061 & 0.728 & 0.648 & 1.101 & 0.725 & 0.018 & 0.040 & -0.003 \\
\hline 4 & 0.671 & 1.061 & 0.859 & 0.691 & 1.101 & 0.881 & 0.020 & 0.040 & 0.022 \\
\hline 5 & 0.862 & 1.112 & 0.928 & 0.882 & 1.137 & 0.951 & 0.020 & 0.025 & 0.023 \\
\hline 6 & 0.862 & 1.196 & 1.149 & 0.882 & 1.199 & 1.175 & 0.020 & 0.034 & 0.026 \\
\hline 7 & 1.776 & 1.376 & 1.502 & $\cdots$ & 1.373 & 1.522 & $\cdots$ & -0.003 & 0.020 \\
\hline 8 & 1.776 & 1.376 & 1.528 & $\cdots$ & 1.373 & 1.524 & $\cdots$ & -0.003 & -0.004 \\
\hline 9 & 1.778 & 1.856 & 1.973 & 1.774 & 1.868 & 1.982 & -0.004 & -0.012 & 0.009 \\
\hline
\end{tabular}

value of the band-structure electronic specific heat $\gamma_{b}$ is $2.06 \times 10^{-4}$ cal mole $\mathrm{e}^{-1} \mathrm{~K}^{-2}$. The experimental values, $\gamma_{e}$, vary from ${ }^{38} 2.0 \times 10^{-4} \mathrm{cal} \mathrm{mole}^{-1}$ $\mathrm{K}^{-2} \mathrm{to}^{39} 3.1 \times 10^{-1} \mathrm{~mole}^{-1} \mathrm{~K}^{-2}$. The experiments by Heiniger et al.$^{40,41}$ gave $\gamma_{e}=2.3 \times 10^{-4} \mathrm{cal} \mathrm{mole}{ }^{-1} \mathrm{~K}^{-2}$, which, if compared to $\gamma_{b}$, implies an apparent electron-phonon enhancement of the thermal effective mass of $12 \%$. This is smaller than the enhancement $(29 \%)$ estimated by McMillan. ${ }^{42}$

\section{Width of $\boldsymbol{d}$ bands. Effects of lattice dilation}

The width of the $d$ bands of a bcc transition metal is often defined as the separation between the $H_{12}$ and $H_{25}$, levels (in the single group repre- sentation) at the symmetry point $H$. The $d$ band width defined in this way is $E\left(H_{8_{+}}^{2}\right)-E\left(H_{8_{+}}^{1}\right)+\frac{1}{2} \xi_{d}$ $=0.791+\frac{1}{2} \times 0.034 \mathrm{Ry}=0.808 \mathrm{Ry}$ when potential $V_{2}$ is applied. One might as well consider $E\left(N_{3}\right)$ $-E\left(H_{12}\right)$ as representing the $d$-band width since $N_{3}$ is $d$ like. This gap is 0.879 Ry. A strict definition of a " $d$-band width" is therefore difficult. This difficulty becomes even more pronounced when comparison is made to $d$-band widths derived from photoemission spectra. Neglecting relativistic effects it is found empirically ${ }^{43}$ that a band of predominantly $l$ character in a closely packed solid extends between the energies $E_{b}$ and $E_{t}$ where $L_{l}\left(E_{b}, R_{\mathrm{WS}}\right)=0$ and $L_{l}\left(E_{t}, R_{\mathrm{WS}}\right) \rightarrow \infty, L_{l}$ being the

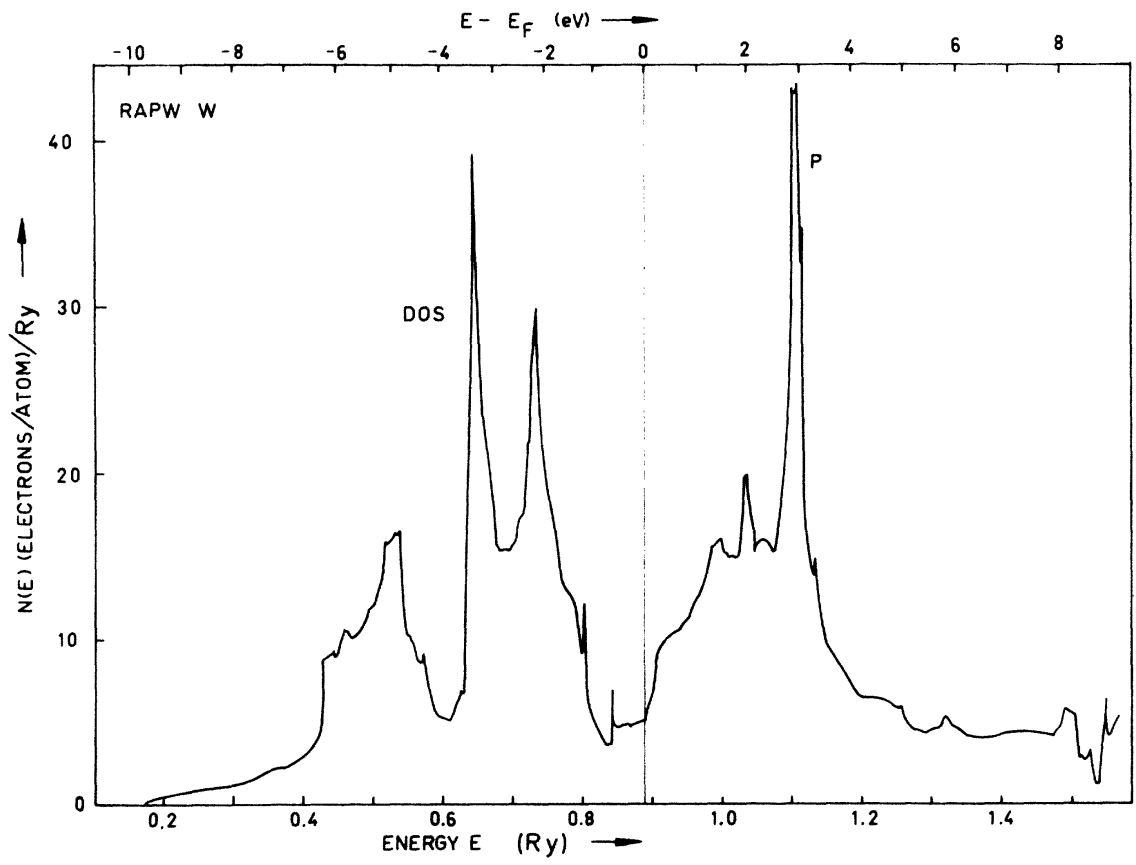

FIG. 3. Density-ofstates calculated by the Gilat-Raubenheimer method in conjunction with interpolation. Potential $V_{2}$. 
logarithmic derivative of the partial wave corresponding to the azimuthal quantum number $l$. The argument $r=R_{\text {ws }}$ indicates that $L_{l}$ is evaluated at the Wigner-Seitz radius. The validity of these Wigner-Seitz rules has been established by Andersen. ${ }^{44,45}$ The parameters $E_{b}$ and $\mathrm{E}_{t}$ for $l=2(d)$ for the potential $V_{2}$ are 0.526 and $1.354 \mathrm{Ry}$, respectively. Thus, the $d$-band width deduced by this simple approach is $0.828 \mathrm{Ry}$, a value close to the one based on the first definition applied above. Since the logarithmic derivatives are very easily obtained, we can quickly calculate the $d$ band width for any potential. Table IV lists the value of $E_{b}$ and $E_{t}$ corresponding to the four potentials $V_{1}, V_{2}, V_{3}$, and $V_{4}$ for $l=2$. In addition, Table IV contains $E_{b}$ for $l=0$ and $1(s, p)$ and a quantity $E_{c}$ for $l=0,1,2$, which gives the approximate "center of gravity" of the band in question. It is determined from $L_{l}$ through ${ }^{44}$

$$
L_{l}\left(E_{c}, R_{\mathrm{ws}}\right)=-(l+1) / R_{\mathrm{ws}} \cdot
$$

It should be noted that the absolute values of the energies listed in Table IV cannot be compared to the band structure shown in Fig. 2 since the band structure calculation includes the relativistic shifts. Figure 4 shows how the width of the $d$ bands in W varies with the lattice parameter $a$. The width $W_{d}$ relative to $W_{d}$ for $a=5.981 \mathrm{a} . \mathrm{u}$. ( $T$ $=300 \mathrm{~K})$ is plotted versus $a$ in Fig. 4(a), and Fig. $4(\mathrm{~b})$ shows the relative decrease of $W_{d}$ as a function of the relative lattice expansion $\Delta a / a(300)$. The dashed lines in both figures give $W_{d}^{\prime} / W_{d}^{\prime}(300)$ $=[a / a(300)]^{-5}$ and $\Delta W_{d}^{\prime} / W_{d}^{\prime}(300)$. These curves are close to the solid lines, demonstrating that the $d$-band width approximately follows an $a^{-5}$ law..$^{46,47}$

The RAPW calculation with $a=6.100$ a. u. yields for the $d$-band width at the symmetry point $H$, $E\left(H_{8+}^{2}\right)-E\left(H_{8_{+}}^{1}\right)+\frac{1}{2} \xi_{d}=0.720+\frac{1}{2} \times 0.034 \mathrm{Ry}=0.737$

Ry. Thus, expansion from the room-temperature

TABLE IV. Band parameters for the potentials $V_{1}$, $V_{2}, V_{3}$, and $V_{4}$ that all are constructed in the same way, but refer to different lattice spacings.

\begin{tabular}{cccccc}
\hline \hline & $V_{1}$ & $V_{2}$ & $V_{3}$ & $V_{4}$ & \\
$a$ & 5.973 & 5.981 & 6.100 & 6.400 & a.u. \\
\hline$l=0(s)$ & & & & & \\
$E_{b}$ & 0.418 & 0.414 & 0.361 & 0.255 & \\
$E_{c}$ & 0.838 & 0.833 & 0.758 & 0.604 & \\
$l=1(p)$ & & & & & \\
$E_{b}$ & 1.069 & 1.063 & 0.972 & 0.788 & \\
$E_{c}$ & 1.870 & 1.862 & 1.737 & 1.472 & \\
$l=2(d)$ & & & & & \\
$E_{b}$ & 0.528 & 0.526 & 0.498 & 0.436 & \\
$E_{c}$ & 0.967 & 0.963 & 0.904 & 0.775 & \\
$E_{t}$ & 1.361 & 1.354 & 1.263 & 1.065 & \\
$E_{t}-E_{b}$ & 0.833 & 0.828 & 0.765 & 0.629 & \\
\hline \hline
\end{tabular}
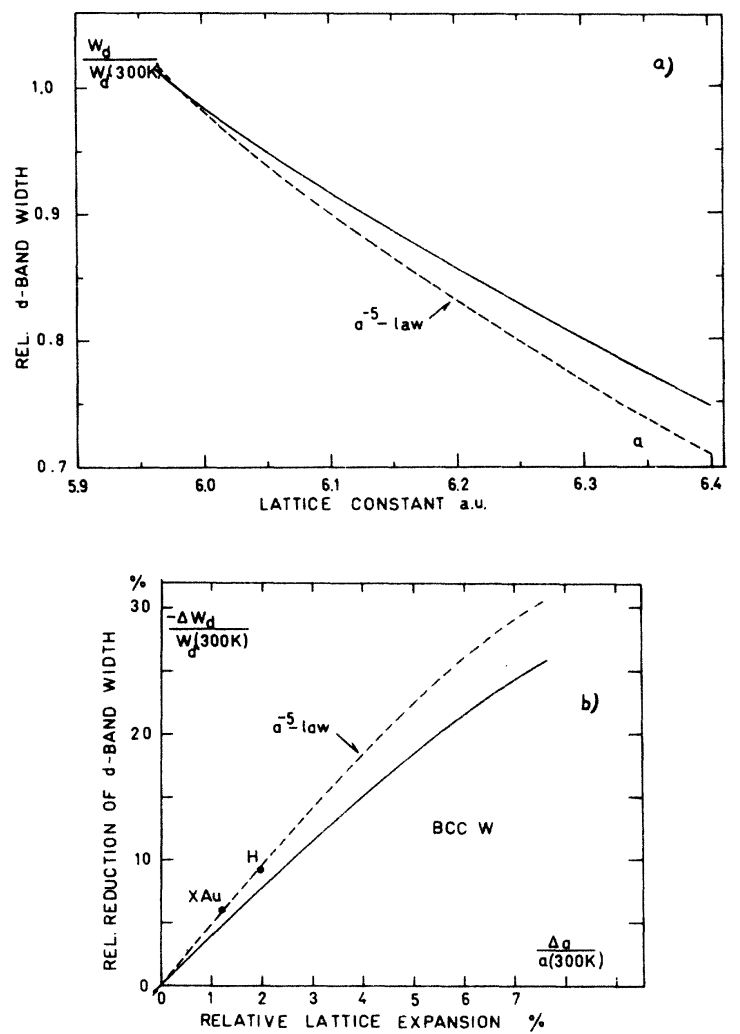

FIG. 4. Relative $d$-band width $W_{d} / W_{d}(300)$ as a function of lattice parameter $a$ (a), and relative decrease of $W_{d}$ versus the relative lattice expansion (b).

lattice to $a=6.100$ a. u. causes this gap to be reduced by 0.071 Ry or $9.2 \%$. Points corresponding to this have been marked $H$ in Fig. 4. The point labeled by $X \mathrm{Au}$ indicating the change in the corresponding energy separation at the symmetry point $X\left[E\left(X_{5}\right)-E\left(X_{3}\right)\right]$ in gold $^{12}$ (fcc) has been included for comparison.

Assuming that the shifts in the energy-band structure due to temperature variation are essentially determined by the thermal expansion of the lattice and not so much by the vibrational smearout of the potential, the temperature responses can be estimated by comparing band structures for different lattice constants. The 4.2 and $300 \mathrm{~K}$ values of $a$ have already been given in Table I. The lattice constant $a=6.100 \mathrm{a}$. u. corresponds to a very high temperature that can only be estimated. The graph given in Ref. 29 or the thermal-expansion coefficient $\beta$ versus temperature has been extrapolated to $T=1300 \mathrm{~K}$ giving $\beta \simeq 10.7 \times 10^{-6}$. This implies that the temperature at which $a=6.100 \mathrm{a} . \mathrm{u}$. is approximately $2150 \mathrm{~K}$, still well below the melting point $(3653 \mathrm{~K})$ of tungsten.

Modulation spectroscopy experiments ${ }^{48-50}$ emphasize Fermi-level transitions and transitions at critical points, where $\nabla_{\overrightarrow{\mathbf{k}}} E_{f}(\overrightarrow{\mathrm{k}})=\nabla_{\overrightarrow{\mathbf{k}}} E_{i}(\overrightarrow{\mathrm{k}})$, i. e., 
initial and final bands are parallel. The first type of transitions may occur over extended regions of $\overrightarrow{\mathrm{k}}$ space. However, since complications arise from Fermi broadening, the present study will be restricted to predictions of thermovariation of a few critical-point transitions. Table $\mathrm{V}$ gives selected critical point energy gaps as calculated for the three potentials $V_{1}, V_{2}$, and $V_{3}$. The largest strain responses are found at $N$ and $P$ for transitions between band 1 and 6 . The $1 \rightarrow 6$ transition at $N$ is of particular interest since the dipole matrix element for this transition is very large. ${ }^{7}$ This gap therefore is expected to show up very strongly in a modulation experiment. ${ }^{51}$ The temperature coefficient for this transition may be obtained from Fig. 5, where the energy gap $E_{g}$ is plotted versus the lattice parameter. For $a=5.981$ a.u. (room temperature), we find $d E_{g} / d a \simeq-2.83$ $\mathrm{eV} / \mathrm{a}$.u. , and with ${ }^{29} \beta=4.7 \times 10^{-6}$ this corresponds to $d E_{\mathrm{g}} / d T=-8 \times 10^{-5} \mathrm{eV} / \mathrm{K}$. It follows from Fig. 5 that the strain response (absolute value) increases strongly with increasing lattice parameter. Further, $\beta$ increases with temperature, and therefore $\left|d E_{g} / d T\right|$ will increase rapidly if the temperature is raised. At $T \sim 1300 \mathrm{~K}$ we have $\beta \sim 10.7 \times 10^{-6}$, and $d E_{g} / d a \simeq-7.3 \mathrm{eV} / \mathrm{a}$. u. giving $d E_{g} / d T \simeq-4.5$ $\times 10^{-4} \mathrm{eV} / \mathrm{K}$. Also the $1 \rightarrow 6$ transition at $P$ becomes very temperature sensitive at high temperatures, $d E_{\mathrm{g}} / d T \sim-5.0 \times 10^{-4} \mathrm{eV} / \mathrm{K}(T \sim 1300 \mathrm{~K})$.

D. Band structures based on $V_{5}, V_{6}, V_{7}$, and $V_{8}$

A reduction of the exchange factor, $\alpha$, produces a broadening of the $d$ bands. ${ }^{10-12}$ This can be anticipated even from the atomic calculation. Since the exchange potential has the same sign as the nuclear potential, a reduction of $\alpha$ will yield atomic wave functions that extend farther from the nucleus. Therefore, when forming the crystal potential, the potential overlaps are increased and so are the tunneling probabilities for the $d$ states.

TABLE V. Selected critical point energy gaps derived from band structures corresponding to three different lattice parameters $a$.

\begin{tabular}{ccccc}
\hline \hline Potential & $V_{1}$ & \multicolumn{1}{c}{$V_{2}$} & \multicolumn{2}{c}{$V_{3}$} \\
\hline $\begin{array}{c}a \\
\text { approximate }\end{array}$ & 5.973 & 5.981 & 6.100 & $\mathrm{a.u}$. \\
$\begin{array}{c}\text { temp. } T \\
1 \rightarrow 6 N\end{array}$ & 12.12 & 300 & 2150 & $\mathrm{~K}$ \\
$2 \rightarrow 6 N$ & 9.31 & 9.28 & 8.51 & $\mathrm{eV}$ \\
$2 \rightarrow 4 N$ & 5.35 & 5.28 & 4.88 & $\mathrm{eV}$ \\
$\begin{array}{c}1 \rightarrow 5 \Delta \\
\mathrm{k}=(4,0,0) \pi / 4 a\end{array}$ & 6.98 & 6.97 & 6.75 & $\mathrm{eV}$ \\
$\begin{array}{c}1 \rightarrow 6 \Delta \\
\mathrm{k}=(5,0,0) \pi / 4 a\end{array}$ & 8.47 & 8.44 & 7.90 & $\mathrm{eV}$ \\
$(2,3) \rightarrow(4,5) P$ & $\cdots$ & 5.93 & 5.45 & $\mathrm{eV}$ \\
$1 \rightarrow 6 P$ & $\cdots$ & 10.26 & 9.36 & $\mathrm{eV}$ \\
\hline \hline
\end{tabular}

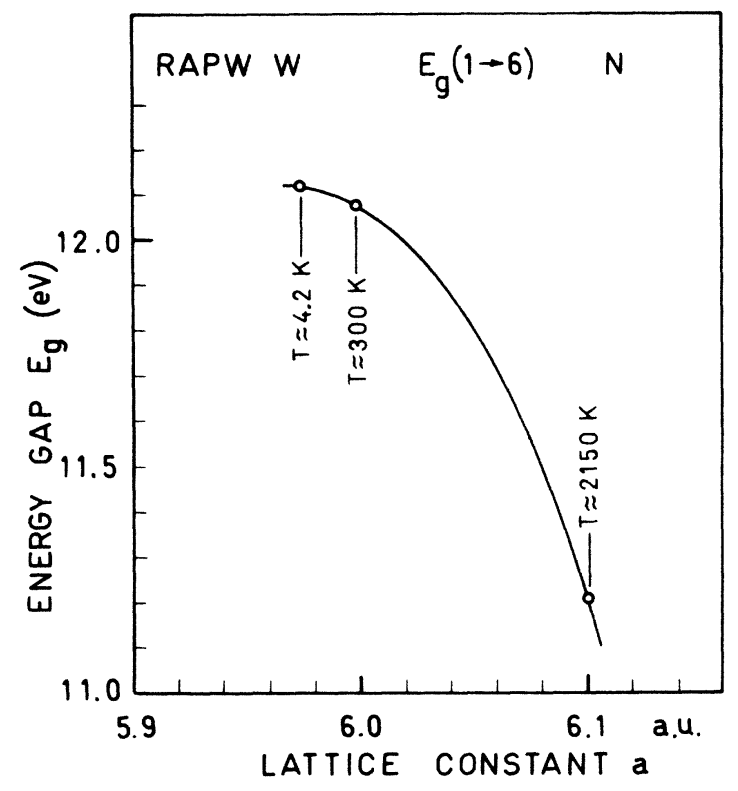

FIG. 5. Energy gap, $E_{g}$, between band 1 (the lowest level) and 6 (counting from below) at $N$ as a function of lattice parameter $a$.

The band structure along the symmetry lines $\Gamma H$, $\Gamma N$, and $\Gamma P$ corresponding to potential $V_{5}$ is shown in Fig. 6. This is still a band structure for a room-temperature lattice constant but $\alpha$ has been reduced to $\frac{2}{3}$ in the self-consistent atomic calculation and in the crystal potential. The electronic configuration is the same as for $V_{2}, 5 d^{4} 6 s^{2}$. The $d$-band parameters $E_{b}, E_{c}$, and $E_{t}(l=2)$ as defined in Sec. II C are 0.599, 1.083, and $1.528 \mathrm{Ry}$, respectively.

The $d$-band width $W_{d}=E_{t}-E_{b}$ is $0.929 \mathrm{Ry}$, i.e., the $d$ bands have broadened by $12 \%$ when compared to the $V_{2}$ results. In particular it should be noted (Fig. 6) that the third band at $N$ is 0.032 Ry below the Fermi level. ${ }^{52}$ If the lattice constant is reduced to the $4.2-\mathrm{K}$ value this separation becomes $0.028 \mathrm{Ry}$. This band is then below $E_{F}$ at $N$ even for $T=4.2 \mathrm{~K}$, and therefore no hole ellipsoids exist. This is in contrast to the experimental results, and we conclude that potential $V_{5}$ does not yield a band structure that is correct in the neighborhood of the Fermi level.

The qualitative features of the energy bands are very similar for all bcc transition metals. Therefore, in view of the results obtained for vanadium by Mattheiss, ${ }^{2}$ it may be expected that the choice of the electronic configuration in the atomic calculation influences the detailed band structure seriously. In Ref. 2 the two configurations $3 d^{4} 4 s^{1}$ and $3 d^{3} 4 s^{2}$ were compared with respect to the band structure of vanadium, and it was found that the $d$-band width derived from the latter potential was 


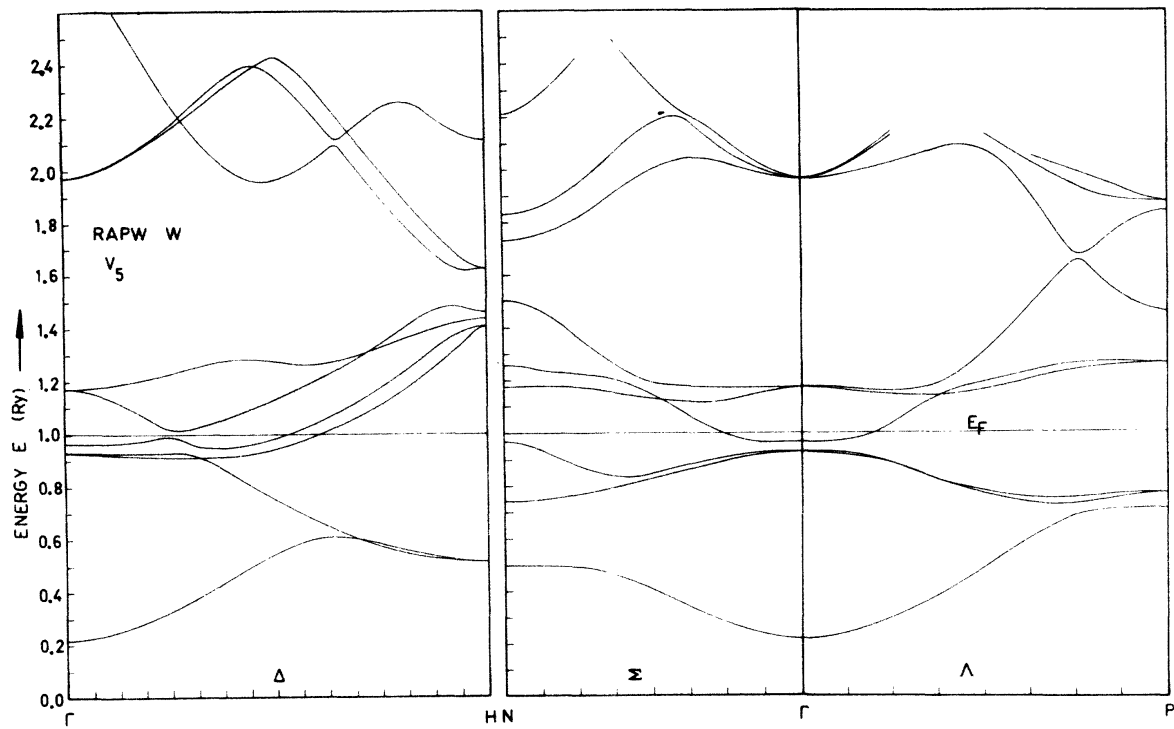

FIG. 6. Band structure of tungsten along the $\Delta, \Sigma$, and $\Lambda$ symmetry lines as calculated from potential $V_{5}$, which is similar to the "best" potential, except for a reduction of the exchange term to $\frac{2}{3}$ of the Slater exchange. only half the value obtained by the $3 d^{4} 4 s^{1}$ potential. An attempt ${ }^{9}$ to reproduce these results for vanadium failed to some extent. The band structures derived from the potentials based on self-consistent atomic calculations for the $3 d^{4} 4 s^{1}$ and $3 d^{3} 4 s^{2}$ configurations differed considerably less than found in Ref. 2. Only by keeping the $3 d^{4} 4 s^{1}$ orbitals fixed and then transferring one $d$ electron to the $s$ shell a potential was constructed that reproduced the very large shifts in the band structure found in Ref. 2. The results for molybdenum ${ }^{9}$ were similar, and the present calculations for tungsten show that also in this case the shifts of the $d$-band widths are small when the configuration is changed self-consistently. The $d$-band width derived from the logarithmic derivatives is 0.846 Ry employing potential $V_{6}$ (configuration $5 d^{5} 6 s^{1}$ ). Comparing this value to that for the $V_{2}$ calculation $\left(5 d^{4} 6 s^{2}\right)$, where the same exchange factor $\alpha$ was included, an increase of only $2.1 \%$ is found. The RAPW energy bands were evaluated along symmetry lines also for the crystal potential $V_{6}$. Furthermore, the effect of reducing the exchange factor $\alpha$ was examined using the $5 d^{5} 6 s^{1}$ configuration. Potential $V_{7}$ includes $\alpha=\frac{2}{3}$ in the atomic (self-consistent) Dirac-Slater calculation and the crystal potential. As expected, this reduction of $\alpha$ gives a further broadening of the $d$-bands $\left(E_{t}-E_{b}=0.950\right.$ Ry, $l=2$ ). If $\alpha$ is reduced to $\frac{2}{3}$ only in the crystal potential $\left(V_{8}\right)$, then the $d$-band width increases even more, namely, to $1.151 \mathrm{Ry}$. Some of the structure parameters corresponding to the potentials $V_{5}-V_{8}$ are compared to those derived for $V_{2}$ in Table VI.

\section{CHOICE OF POTENTIAL}

The result of the discussion in this section has to some extent been anticipated. The band struc- ture based on the potential with full Slater exchange and based on the atomic calculation for the groundstate configuration $\left(V_{2}\right)$ has been described extensively. The reason is that this band structure seems to provide the best fit to the experimental room-temperature data currently available. In this section the choice will be further justified by comparing a calculated optical spectrum to the experimental results of Nomerovannaya et al.$^{17}$ Additional support for the choice of potential is given by the fact that only the low-temperature analog, $V_{1}$ yields Fermi-surface dimensions in agreement with experimental results.

Since the Fermi level is close to the center of the $d$ bands only small changes in the band structure around the Fermi surface are produced by the modifications of the potential. Nevertheless, small shifts influence the Fermi surface near $N$, and this sensitivity permits a selection of reasonable potentials. Experiments show ${ }^{13}$ that hole elliposids exist around $N$, i.e., band 3 must be above $E_{F}$ at this point. Further, the onset of interband transitions from band 3 to band 7 near $N$ appears to occur at $10.1-\mathrm{eV}$ photon energy. ${ }^{23}$ These quantities are summarized in the last two lines of Table VI for potentials $V_{2}, V_{5}, V_{6}$, and $V_{7}$. Apparently only the band structures based on $V_{2}$ and $V_{6}$ predict the existence of the hole ellipsoids. The ellipsoids obtained from the $V_{6}$ calculation are very small, and are in fact too small as will be shown below. The calculated threshold for the $3 \rightarrow 7$ transitions around $N$ varies from 10.4 to $10.9 \mathrm{eV}$, all somewhat exceeding the value deduced from photoemission experiments. ${ }^{23}$ The observed threshold energy may, ${ }^{23}$ however, be slightly low due to errors in crystal alignment and due to the finite acceptance angle of the photoemission analyzer used in these experiments. 
TABLE VI. $d$-band parameters and important energy separations calculated from five different potentials $V_{2}, V_{5}, V_{6}, V_{7}$, and $V_{8}$ all corresponding to the room-temperature lattice constant. $E_{c 1}$ and $E_{c 2}$ denote the $d$-band center at $\Gamma$ and $H$, respectively; i. e. , $E_{c 1,2}=\frac{1}{5}\left[E\left(7^{+}\right)+2 E\left(8^{+1}\right)+2 E\left(8^{+2}\right)\right]$. The other parameters are defined in the text.

\begin{tabular}{ccccccc}
\hline \hline Potential & \multicolumn{1}{c}{$V_{2}$} & $V_{5}$ & $V_{6}$ & $V_{7}$ & $V_{8}$ & \\
\hline$\alpha_{a}, \alpha_{c}$ & 1,1 & $2 / 3,2 / 3$ & 1,1 & $2 / 3,2 / 3$ & $1,2 / 3$ & \\
Config. & $5 d^{4} 6 s^{2}$ & $5 d^{4} 6 s^{2}$ & $5 d^{5} 6 s^{1}$ & $5 d^{5} 6 s^{1}$ & $5 d^{4} 6 s^{2}$ & \\
$E_{b}(l=2)$ & 0.527 & 0.599 & 0.542 & 0.620 & 0.736 & $\mathrm{Ry}$ \\
$E_{c}(l=2)$ & 0.963 & 1.083 & 0.987 & 1.115 & 1.316 & $\mathrm{Ry}$ \\
$E_{t}(l=2)$ & 1.354 & 1.528 & 1.388 & 1.570 & 1.877 & $\mathrm{Ry}$ \\
$E_{t}-E_{b}$ & 0.828 & 0.929 & 0.846 & 0.950 & 1.141 & $\mathrm{Ry}$ \\
$E_{F}$ & 0.889 & 1.001 & 0.905 & 1.025 & & $\mathrm{Ry}$ \\
$E_{c 1}$ & 0.904 & 1.030 & 0.929 & 1.082 & & $\mathrm{Ry}$ \\
$E_{c 2}$ & 0.929 & 1.054 & 0.956 & 1.085 & & $\mathrm{Ry}$ \\
$E\left(H_{8+}^{2}\right)-E\left(H_{8+}^{1}\right)$ & 0.791 & 0.891 & 0.810 & 0.916 & & $\mathrm{Ry}$ \\
$E\left(\Gamma_{8+}^{2}\right)-E\left(\Gamma_{8+}^{1}\right)$ & 0.232 & 0.240 & 0.238 & 0.242 & & $\mathrm{Ry}$ \\
$E_{F}-E\left(\Gamma_{6+}\right)$ & 0.716 & 0.782 & 0.726 & 0.795 & & $\mathrm{Ry}$ \\
$E_{F}-E(3)$ at $N$ & -0.014 & 0.033 & -0.005 & 0.045 & & $\mathrm{Ry}$ \\
$\hbar \omega_{\text {ons }}$ & 10.6 & 10.4 & 10.9 & 10.4 & & $\mathrm{eV}$ \\
$(3 \rightarrow 7$ at $N)$ & & & & & \\
\hline \hline
\end{tabular}

Fermi-surface (see Fig. 7) dimensions calculated from the potentials $V_{1}, V_{2}, V_{5}, V_{6}$, and $V_{7}$ are listed together with results of the size-effect measurements by Boiko et al. ${ }^{13}$ in Table VII. Since only $V_{1}$ refers to the temperature $(T \simeq 4.2 \mathrm{~K})$ at which the actual experiment was carried out, and because of the slight uncertainty in the exact positioning of the Fermi level, ${ }^{52}$ the comparison between the experiment and the calculations should be made by means of the ratios of dimensions in Table VII rather than by the dimensions themselves. Also, possible experimental errors in the sample thickness determination are cancelled in the ratios. Table VII shows ${ }^{52}$ that the potentials $V_{1}$ and $V_{2}$ give the best agreement with the experiment. The ratios between the Fermi-surface dimensions change only slightly if the room-temperature potentials are replaced by potentials constructed in the same way but for the 4.2-K lattice. This is apparent from the first two columns of Table VII. Therefore, the experimental ratios would not be reproduced even if $V_{5}, V_{6}$, and $V_{7}$ were replaced by the corresponding low-temperature potentials. It is therefore concluded that $V_{2}$ is the best potential at room temperature as far as energy eigenvalues near $E_{F}$ are concerned.

It remains to establish whether the band structure obtained from $V_{2}$ is also correct away from the Fermi level. For this purpose it is pertinent to compare the calculated optical interband transition energies with the results of reflectance measurements. As far as static-optical measurements are concerned we are restricted to a comparison of functions such as the imaginary part $\epsilon_{2}(\omega)$ of the dielectric function, which integrate over all transitions throughout the entire Brillouin zone rather than specific energy gaps. The joint density - of states function

$$
\begin{aligned}
J(\hbar \omega) \propto & \sum_{i, f} \int d^{3} k \delta\left(E_{f}(\overrightarrow{\mathrm{k}})-E_{i}(\overrightarrow{\mathrm{k}})-\hbar \omega\right) \\
& \times f\left(E_{i}(\overrightarrow{\mathrm{k}})\right)\left[1-f\left(E_{f}(\overrightarrow{\mathrm{k}})\right)\right],
\end{aligned}
$$

which sums the transitions at the photon energy $\hbar \omega$ from occupied initial states $E_{i}(\vec{k})$ to empty final states $E_{f}(\vec{k})$ is proportional to $\epsilon_{2}(\omega) \omega^{2}$-if the dipole matrix elements are assumed to be constant. The Fermi functions $f$ and $(1-f)$ in (4) ensure that the initial states are occupied and the final states empty. The APW calculation by Petroff and Viswanathan ${ }^{7}$ indicated the matrix elements to vary strongly with $\vec{k}$ and energy, but nevertheless the simple model assuming constant transition probabilities will be adopted here.

The joint density-of-states function was calculated in the same way as $N(E)$, i. e., by a combina$\operatorname{tion}^{12}$ of an interpolation scheme and the GilatRaubenheimer method. The derived $\epsilon_{2}(\omega)$ profile



FIG. 7. Fermi-surface contours for tungsten (qualitative sketch). 
TABLE VII. Fermi-surface dimensions as calculated from the potentials $V_{1}, V_{2}$, $V_{5}, V_{6}$, and $V_{7}$. Note that only $V_{1}$ refers to helium temperature. Therefore, for $V_{5}, V_{6}$, and $V_{7}$, most eniphasis should be on a comparison and the experimental ratios. No ellipsoids exist at $N$ when $V_{5}$ and $V_{7}$ are used (see, however, Ref. 52).

\begin{tabular}{|c|c|c|c|c|c|c|}
\hline $\begin{array}{c}\alpha_{a}, \alpha_{c} \\
\text { Config. } \\
\text { temp. } T .\end{array}$ & $\begin{array}{c}V_{1} \\
1,1 \\
5 d^{4} 6 s^{2} \\
4.2 \mathrm{~K}\end{array}$ & $\begin{array}{c}V_{2} \\
1,1 \\
5 d^{4} 6 s^{2} \\
300 \mathrm{~K}\end{array}$ & $\begin{array}{c}V_{5} \\
2 / 3,2 / 3 \\
5 d^{4} 6 s^{2} \\
300 \mathrm{~K}\end{array}$ & $\begin{array}{c}V_{6} \\
1,1 \\
5 d^{5} 6 s^{1} \\
300 \mathrm{~K}\end{array}$ & $\begin{array}{c}V_{7} \\
2 / 3,2 / 3 \\
5 d^{5} 6 s^{1} \\
300 \mathrm{~K}\end{array}$ & $\begin{array}{c}\text { Experiment } \\
\text { Ref. } 13 \\
4.2 \mathrm{k}\end{array}$ \\
\hline \multicolumn{7}{|l|}{$\begin{array}{c}\text { Electron } \\
\text { jack }\end{array}$} \\
\hline$J_{\Gamma H}$ & 1.018 & 1.016 & 1.022 & 1.029 & 1.016 & $1.09 \pm 0.02 \AA^{-1}$ \\
\hline$J_{\Gamma N}$ & 0.427 & 0.409 & 0.365 & 0.341 & 0.334 & $0.46 \pm 0.01 \AA^{-1}$ \\
\hline$J_{\Gamma P}$ & 0.389 & 0.372 & 0.334 & 0.347 & 0.322 & $0.41 \pm 0.01 \AA^{-1}$ \\
\hline$J_{\Gamma N} / J_{\Gamma P}$ & 1.10 & 1.10 & 1.09 & 0.98 & 1.04 & 1.12 \\
\hline$J_{\Gamma N} / J_{\Gamma H}$ & 0.42 & 0.40 & 0.36 & 0.33 & 0.33 & 0.42 \\
\hline $\begin{array}{c}\text { Hole } \\
\text { ellipsoids } \\
\text { around } N\end{array}$ & & & & & & \\
\hline$E_{N \Gamma}$ & 0.124 & 0.087 & 0 & 0.050 & 0 & $0.143 \pm 0.004 \AA^{-1}$ \\
\hline$E_{N P}$ & 0.173 & 0.120 & 0 & & 0 & $0.195 \pm 0.006 \AA^{-1}$ \\
\hline$E_{N H}$ & 0.106 & 0.075 & 0 & & 0 & $0.121 \pm 0.004 \AA^{-1}$ \\
\hline$E_{N \Gamma} / E_{N P}$ & 0.72 & 0.72 & & & & 0.73 \\
\hline$E_{N \Gamma} / E_{N H}$ & 1.17 & 1.16 & & & & 1.18 \\
\hline \multicolumn{7}{|c|}{$\begin{array}{l}\text { Hole octa- } \\
\text { hedron }\end{array}$} \\
\hline$O_{H \Gamma}$ & 0.779 & 0.773 & 0.795 & & & $0.75 \pm 0.015$ \\
\hline$O_{H N}$ & 0.610 & 0.604 & & & & $0.60 \pm 0.01$ \\
\hline$O_{H P}$ & 0.477 & 0.478 & & & & $0.50 \pm 0.001$ \\
\hline$O_{H \Gamma} / O_{H N}$ & 1.28 & 1.28 & & & & 1.25 \\
\hline$O_{H N} / O_{H P}$ & 1.30 & 1.30 & & & & 1.20 \\
\hline
\end{tabular}

is shown in Fig. 8, together with the experimental trace obtained by Nomerovannaya et al. ${ }^{17}$ The calculated spectrum has been scaled vertically such that it coincides with the measured $\epsilon_{2}$ function at one single point $\hbar \omega=0.5 \mathrm{eV}$. No energy shift or other adjustments were made. In view of the crude approximation concerning the matrix elements it is not surprising that the line shapes are not well reproduced. Nevertheless, in the energy range where the measured $\epsilon_{2}$ function is available, agreement is found between the spectral positions of the pronounced elements of structure in the two curves. It may therefore be concluded that the band structure (potential $V_{2}$ ) shows good agreement with the optical experiment for excitation energies up to $5 \mathrm{eV}$. At present, only photoemission experiments provide data at higher photon energies for comparison. The accuracy of the band structure in this range will be discussed in detail subsequently. ${ }^{23}$

It is generally not possible ${ }^{12}$ to relate peaks in $\epsilon_{2}(\omega)$ to specific critical points in the band structure in a unique way, but to some extent we can analyze the various contributions to each peak with respect to the band pairs and regions in $\vec{k}$ space involved. For that purpose each of the partial joint density-of-states functions ${ }^{12} J_{i f}(\hbar \omega)$ was plot- ted, $J_{i f}$ being the contribution to the joint density of states from transitions between the initial band ${ }^{33}$ $i$ and the final band $f$. In the range of photon energies applied in the experiment, i.e., for $\hbar \omega<5 \mathrm{eV}$, $\epsilon_{2}$ has contributions from as many as 10 band pairs, namely, $(i, f)=(1,3),(2,3),(2,4),(2,5),(2,6)$, $(3,4),(3,5),(3,6),(4,5)$, and $(4,6)$. These functions are shown in Fig. 9. No interband transitions occur below $0.35 \mathrm{eV}$, where the onset of $4-5$ transitions is found. This edge corresponds to the SO gap between band 4 and 5 at $\Delta$ (Fig. 2). The structure found experimentally ${ }^{17}$ at $0.08 \mathrm{eV}$ therefore cannot be explained in terms of interband transitions in the present band model. Since the present band model has been shown to be accurate near $E_{F}$ the tentative assignment made in Ref. 17 of this structure to transitions between band 3 and band 4 near a $G$ point cannot be supported. Following the calculation such transitions should occur for $\hbar \omega$ slightly above $0.5 \mathrm{eV}$. The $3 \rightarrow 4$ transitions start at $\hbar \omega=0.50 \mathrm{eV}$ and the edge corresponds to the parallel-band transitions between the se two SO-split bands in a region around the $\Delta$ line. This means that the present calculation of the $3-4$ and $4-5$ onset edges agrees well with the interpretation in Ref. 17 of the structures in the conductivity ${ }^{53}$ observed at 0.33 and $0.4 \mathrm{eV}$, 




FIG. 8. $\epsilon_{2}(\omega)$ spectrum obtained (full line) from the joint density-of-states function derived from the $V_{2-}$ band structure. The broken curve was obtained from the experimental $n(\omega)$ and $k(\omega)$ values given in Ref. 17, $n$ and $k$ being the real and imaginary parts of the refractive index.

with the reservation that a peak in the conductivity does not give the spectral position of an edge. The same band pairs $(3,4),(4,5)$ are also responsible for the structure around $1 \mathrm{eV}$. It is interesting to note that even the small peak on the $\epsilon_{2}(\omega)$ function observed at $1.35 \mathrm{eV}$ is present in the calculated curve. At higher energies, five clear structure elements labeled $A, B, C, D$, and $E$ in Fig. 8 appear in the calculated $\epsilon_{2}$ trace. Although the matrix elements were assumed to be constant in the calculation it appears reasonable to identify these five structure elements with those labeled $A^{\prime}, B^{\prime}$, $C^{\prime}, D^{\prime}$, and $E^{\prime}$ in the experimental trace. The structure $F^{\prime}$ between $C^{\prime}$ and $D^{\prime}$ in the experimental curve is tentatively related to the shoulder on the low-energy side of peak $D$ in the calculated $\epsilon_{2}$ function. With this assignment it follows from an examination of the partial joint density-of -states functions (Fig. 9) that $A^{\prime}(A)$ is due to a large number of transitions between band 4 and 5 around 1. $7 \mathrm{eV}$. The maxima $B$ and $B^{\prime}$ coincide at $2.2 \mathrm{eV}$, and this structure is essentially due to transitions between band 4 and $5 . C^{\prime}(C)$ at $2.4 \mathrm{eV}$ contains the sharp peak of $J_{46}$. The structure in $J_{24}$ at this energy will be enhanced in the $\epsilon_{2}$ spectrum due to the factor $1 / \omega^{2}$. The low-energy shoulder of the $D$ hump that was related to the peak $F^{\prime}$ at $2.75 \mathrm{eV}$ most likely is due to $3 \rightarrow 5$ transitions. The first distinct peak at $3.0 \mathrm{eV}$ in the $D$ hump is due to $2 \rightarrow 5$ transitions (note the scaling of the $J_{i f}$ curves given in the right-hand corner of Fig. 9). This is not expected to show up clearly in the experimental trace since it reflects forbidden $d-d$ transitions near $\Gamma$. The major contributions to $D$ come from the $3 \rightarrow 4$ transitions. A strong peak extends from 3.15 to $3.30 \mathrm{eV}$, where two clear Van Hove singularities are in $J_{34}$. The singularity at $3.30 \mathrm{eV}$ is related to parallel-band transitions at a critical point of type $M_{2}$ at the $\Sigma$ line. This gap is increased to $3.80 \mathrm{eV}$ if potential $V_{6}$ is applied and thus $D$ would in that case be located at a spectral position which is above the experimental $D^{\prime}$. Since the potentials $V_{5}, V_{7}$, and $V_{8}$ all give $d$ bands that are wider than those obtained from $V_{6}$ it follows that the discrepancy between calculation and experiment becomes even worse when those potentials are used. The weak structure $E^{\prime}(E)$ at $4.25 \mathrm{eV}$ is ascribed to $2 \rightarrow 4$ transitions.

It may be concluded that only one of the potentials examined agrees with experimental results. This is $V_{2}$, i.e., the potential based on the ground state configuration in the atom and with inclusion of full Salter exchange. The band structure derived from this potential agrees with experimental results at the Fermi level $E_{F}$ and at least up to $5 \mathrm{eV}$ away from $E_{F}$. The other models fail in both respects. Although the band structures so far have not been tested beyond $E_{F}+5 \mathrm{eV}$ we decided to apply the band structure selected as the best one from the above criteria in an analysis of directional photoemission experiments. ${ }^{23}$

\section{PHOTOEMISSION SPECTRA}

The relevance of the calculations presented in this section to photoemission experiments ${ }^{23}$ rests on the assumption that surface and volume contributions to the photoemission spectra can be separated. The validity of such a separation into one part that solely reflects bulk properties and another related to surface properties is not entirely justifiable since there may be a gradual transition between the two types of mechanisms when the surface is approached from the bulk. If, for instance, bulklike excitation mechanisms apply even close to the surface the dipole matrix elements may be influenced by the detailed shape of the surface potential function. Since these matrix elements constitute a squared factor in the transition probability it is obvious that cross terms be- 

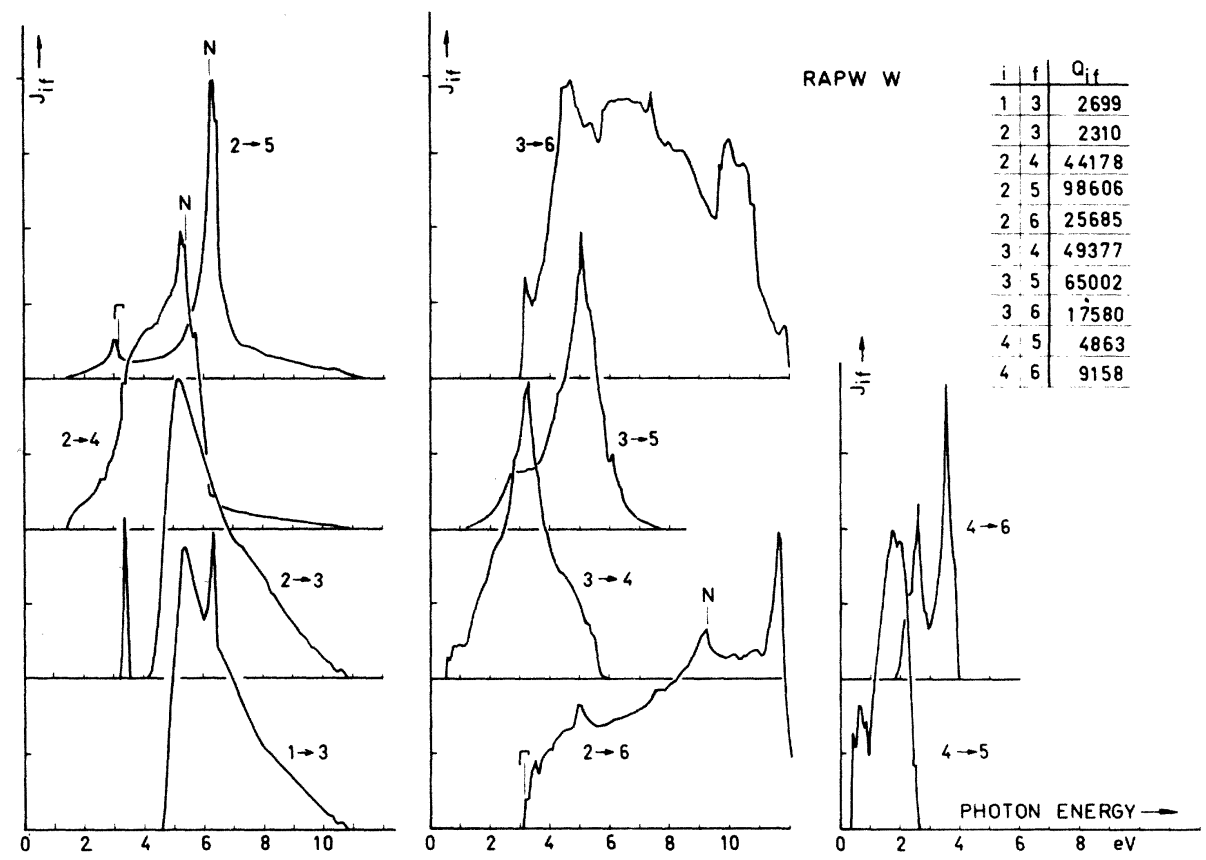

FIG. 9. Partial joint density-of-states functions. $J_{i f}$ gives the number of possible direct transitions $(i \rightarrow f)$ from that part of band $i$ which is below the Fermi level to that part of band $f$ which is above $E_{F}$. The band energies are numbered with increasing energy. All curves have been scaled to the same maximum value. The number $Q_{i f}$ gives the maximum value of $J_{i f}$ (arbitrary units). tween crystal and surface potentials exist and a strict decoupling of the two cannot be performed. However, in the experimental work of Ref. 23 it is shown that a phenomenological separation appears to be justified in the case of tungsten.

In this section various calculations related to bulk and surface contributions to photoemission spectra are presented. It is left to Ref. 23 to select what parts are most likely to contribute to the experimental traces. Full solid-angle spectra as well as spectra for particular emission directions relative to low-index crystal faces are presented.

\section{A. Bulk contributions}

Experimentally recorded full solid-angle photoemission spectra have often been successfully interpreted in terms of simple models assuming that mainly bulk electronic properties are reflected. These models consider the photoemission process as consisting of three steps; excitation from an initial-band state to a final-band (i.e., bulk) state, transport of the hot electron toward the surface, and finally emission through the surface. Two different types of excitation processes have been considered, namely "nondirect" and "direct" transitions. In the "nondirect" model ${ }^{54-56}$ it is assumed that $\overrightarrow{\mathrm{k}}$ conservation is not important during the excitation. The "direct" model requires conservation of crystal momentum, i. e., the reduced $\vec{k}$ vector of the final state is the same as that of the initial state, apart from the negligible small wave vector of the photon. Early photoemission data for copper and silver were explained ${ }^{55}$ in terms of the nondirect model. More recent experiments with improved resolution demonstrated that at least for gold and copper the direct model should give more satisfactory agreement. ${ }^{19,21,57-63}$

If $\overrightarrow{\mathrm{k}}$ conservation is unimportant, the number of electrons excited from the initial energy $E_{i}^{0}$ by photon with energy $\hbar \omega, D\left(E_{i}^{0}, \hbar \omega\right)$ can be written

$$
D\left(E_{i}^{0}, \hbar \omega\right) \propto \rho_{i}\left(E_{i}^{0}\right) \rho_{f}\left(E_{i}^{0}+\hbar \omega\right),
$$

where $\rho_{i}$ and $\rho_{f}$ are the initial and final "optical density of states." 54,56 They differ from the usual density of states $N(E)$, because of the included transition probabilities and Fermi factors and due to many-body effects. In contrast the energy distribution in the direct model including $\overrightarrow{\mathrm{k}}$-dependent matrix elements is given by

$$
\begin{aligned}
D\left(E_{i}^{0}, \hbar \omega\right) \propto & \sum_{i, f} \int d^{3} k \delta\left(E_{f}(\overrightarrow{\mathrm{k}})-E_{i}(\overrightarrow{\mathrm{k}})-\hbar \omega\right) \\
& \times \delta\left(E_{i}^{0}-E_{i}(k)\right)\left|M_{i f}\right|^{2} \\
& \times f\left(E_{i}^{0}\right)\left[1-f\left(E_{i}^{0}+\hbar \omega\right)\right] .
\end{aligned}
$$

The first $\delta$ function picks out the energy differences in the band structure that equals the photon energy, whereas the second ensures that only those transitions that have an initial energy $E_{i}^{0}$ are counted. The factor $\left|M_{i f}\right|^{2}$ is the transition probability. The function $D\left(E_{i}^{0}, \hbar \omega\right)$ as defined by Eq. (6) would, if the dipole matrix elements were constant, essentially be the energy distribution of joint density of states (EDJDOS), referring to initial energy.

If the variation of the matrix elements with $\vec{k}$ and energy is neglected the functions $D\left(E_{i}^{0}, \hbar \omega\right)$ are 



FIG. 10. Energy distributions $D\left(E_{i}, \hbar \omega\right)$ of photoexcited electrons. The left-hand part shows $D$ in the nondirect model, whereas the direct model distributions are shown in the right-hand section.

easily obtained from the band structure. Figure 10 shows the distributions corresponding to the two models, the nondirect (a) and the direct (b). All curves have been folded by a Lorentzian of 0. 3-eV full half-width. The dashed line in Fig. $10(a)$ shows the density of initial states $N(E)$. It is seen that the $D\left(E_{i}^{0}, \hbar \omega\right)$ functions for both direct and nondirect models differ considerably from $N(E)$. Even in the nondirect model many features appear in the $D\left(E_{i}^{0}, \hbar \omega\right)$ functions due to structure in the density of final states, which is particularly pronounced in tungsten. The vertical bar indicates structure related to the strong peak $P$ in Fig. 3 . The EDJDOS functions, i. e., $D\left(E_{i}^{0}, \hbar \omega\right)$ corresponding to direct transitions change their shape drastically as the photon energy is varied. Only at some particular photon energies, say around 10 $\mathrm{eV}$, it is clear that structure is found which is a common feature of $N(E)$ and the two $D$ functions. To some extent final-state structure in Fig. 10(b) has been attenuated ${ }^{8,11,18}$ by an extra factor $\left|\overrightarrow{\mathbf{v}}_{\overrightarrow{\mathbf{k}}}\right|$ $=\left|\vec{\nabla}_{\mathfrak{k}} E_{f}(\vec{k})\right|$ in the integrand of Eq. (6). This factor was included to take account of the final-state group velocity effects on the transport of excited electrons out of the crystal.

If the distributions $D\left(E_{i}^{0}, \hbar \omega\right)$ shown in Fig. 10 are multiplied by a threshold function, ${ }^{64}$ emission spectra as shown in Fig. 11 are obtained. These spectra do not contain any directional effects, and should be related to large acceptance angle measurements on "true" polycrystalline samples. It is important to note that a full-angle emission mea- surement on a single crystal does not correspond to such spectra since the internal excitation cone angle is smaller than $180^{\circ}$ due to the finite work function. This may be seen by comparing Fig. 11(b) to Fig. 12, where, in the latter, the directmodel spectra for $\hbar \omega \geq 10.2 \mathrm{eV}$ from the three low-index faces covering all external angles are shown. Comparing the 10.2-eV spectra in Figs. 11 (b) and 12 it is seen that the broad hump on the low-energy side $(-6$ to $-4 \mathrm{eV})$ has disappeared in the (110) and (111) traces. The two main peaks are pronounced in all curves. The (110) energydistribution curve (EDC) as measured by Baker and Eastman ${ }^{65}$ for $\hbar \omega=16.8 \mathrm{eV}$ is shown as the broken curve in Fig. 12. The spectral position of the peaks in the experimental trace agrees well with the predictions from the direct model calculation. However, a comparison to the dashed curve in Fig. 10 demonstrates that the experimental EDC agrees equally well with the overall broadened density of states, $N(E)$. Comparison to the experiment in this case therefore provides no critical test of the emission model and the band structure in the high-energy region.

Photoemission spectra will to some extent reflect the functions $D\left(E_{i}^{0}, \hbar \omega\right)$ defined above. However, the measured spectrum will strongly depend on the experimental arrangement. As a first step, we consider the case in which the calculations are to be compared to experiments where the excitation is as general as possible, i.e., the light is unpolarized at oblique incidence on the sample and 



FIG. 11. Energy-distribution curves (EDC's) referred to initial state energy for electrons emitted from a polycrystalline sainple. The nondirect-model EDC's are shown in (a), and spectra calculated within the direct model are shown in (b). spread over a large cone of incidence angles. In the free-electron model, normally incident light does not produce any photoemission.667 Further, we may choose an experiment that detects only electrons in a narrow cone, such that for single crystal samples the recorded spectrum depends sensitively on the emitting crystal plane and on the acceptance angle of the analyzer system.

Therefore, in addition to the results shown in Figs. 11 and 12 , calculations will be presented that are related to two different types of measurement technique; one in which all electrons emitted within a certain cone around the surface normal are counted, and the other in which only the electrons emitted in directions between two cones defined by the angles $\phi+\Delta \phi$ and $\phi-\Delta \phi$ measured from the sample normal are detected. The latter experimental arrangement is similar to that used by Gustafsson, Nilsson, and Wallden ${ }^{68}$ in their measurements on silver. The first type of measurement technique, or the second with $\phi=0$ is used in the experiments described in Ref. 23.

If the crystal lattice is perfect in the bulk and at the surface in the sense that the periodicity in directions parallel to the surface is the same as in the bulk, then the component of the wave vector parallel to the surface is conserved during the electron emission process. ${ }^{67,69}$ Note in this context that it is the wave vector of the final excited state in the crystal that should be matched in this way to the external momentum. The present calculations do not include the so-called "secondary cones, "66 i. e., contributions due to an accurate matching to the external wave functions that requires the inclusion of components of the interior wave functions corresponding to $\overrightarrow{\mathrm{k}}+\overrightarrow{\mathrm{G}}_{i}, \overrightarrow{\mathrm{G}}_{i}$ being reciprocal-lattice vectors. The omission of such umklapp processes may be particularly serious for the calculated (111) spectra. As shown in Fig. 1 , a reciprocal-lattice vector of the $(1,1,0)$ type connects points on the continuation of a $\Lambda$ line outside the Brillouin zone to points on a $P H$ line. The present model where all umklapp processes are neglected is further discussed in Ref. 23.

The approach which is followed here to obtain the angular distribution of the emitted electrons is then straight forward. The kinetic energy $E_{\mathrm{k} i n}$ of an electron with momentum $\overrightarrow{\mathrm{p}}$ outside the sample is $p^{2} / 2 m$, and for a certain kinetic energy and emission angle $\phi$-see Fig. 13-the parallel momentum $\overrightarrow{\mathrm{p}}_{n}$ is obtained by projection on the surface plane. The kinetic energy is related to the initialstate energy $E_{i}^{0}$ through

$$
E_{i}^{0}+\hbar \omega=E_{F}+W+E_{\mathrm{k} \text { in }},
$$

where $W$ is the work function, and $E_{i}^{0}$ and $E_{F}$ are measured from a common reference. The functions $D\left(E_{i}^{0}, \hbar \omega\right)$ defined earlier give the total number of electrons excited from the initial energy level $E_{i}^{0}$ to final states with energy $E_{i}^{0}+\hbar \omega$. Only a part of these electrons appear outside the crystal with momenta $\vec{p}$ on the cone defined by $\phi$. Only those transitions for which the crystal final-state energy satisfies $\mathrm{Eq} .(7)$ and the $\overrightarrow{\mathrm{k}}$ vector has a 



FIG. 12. Full angle direct EDC's for (100), (110), and (111) faces for the photon energies $10.2,10.9$, 11.6 , and $16.8 \mathrm{eV}$. The spectra were calculated in the same manner as those shown in Fig. 14. The broken curve represents the experimental trace obtained by Baker and Eastman (Ref. 65) at $16.8 \mathrm{eV}$.

parallel component equal to $\left|\vec{p}_{\| 1}\right|$ in magnitude can contribute to the spectrum detected in the directions defined by $\phi$ and corresponding to a specific initial state energy.

Figures 14 and 15 show the emission spectra calculated within the direct model for different acceptance angles and crystal faces [(100), (110), (111)]. For each face the experimental ${ }^{23}$ value of the work function was used, namely, $4.3 \mathrm{eV}$ for (100) and (111) and $5.1 \mathrm{eV}$ for the (110) face. In Fig. 14 the emission curves for certain acceptance angles are shown, simulating an experiment where only those electrons emitted within a cone are detected. Calculations for only one photon energy $(\hbar \omega=11.6 \mathrm{eV})$ are shown as illustration, but they have been performed for several ${ }^{70}$ energies ranging from 10 to $16.8 \mathrm{eV}$. The spectra ${ }^{71}$ in Fig. 14 vary drastically with the analyzer acceptance angle. Although a half-cone angle of $\phi=30^{\circ}$ might be considered large, the spectrum is in some cases entirely different from the full-angle trace (compare, for instance, the calculated emission from the (100) face in Figs. 12 and 14). This means that a comparison between an experiment with restricted but unspecified acceptance angle to a full angle theoretical calculation is meaningless. The fact that the (111) spectra seem to be rather insensitive to the change in acceptance angle (Fig. 14) is particular to the photon energy $\hbar \omega=11.6 \mathrm{eV}$, where the strong ${ }^{71}-3.5-\mathrm{eV}$ peak dominates. The maximum value of the (111) curve for $\phi=15^{\circ}$ is in arbitrary units 6771 , whereas the largest (100) peak is only 629 on the same scale. Fine structure is therefore strongly suppressed in the (111) curves.
If the experimental arrangement is changed such that only electrons emitted in directions between the cones with half-opening angles $\phi-\Delta \phi$ and $\phi+\Delta \phi$ are counted, a further restriction is imposed on the region in $\overrightarrow{\mathrm{k}}$ space that contributes. Therefore, the shape of the recorded spectra will be different again from those in Fig. 14. This is clear from a comparison between the calculations presented in Figs. 14 and 15, especially for $\phi=45^{\circ}$ relative to the (100) normal, which is entirely different for the curves shown in Figs. 14 and 15.

We now turn our attention to the emission in directions that are normal $(\phi=0)$ to the three low index planes. In the direct model such a spectrum
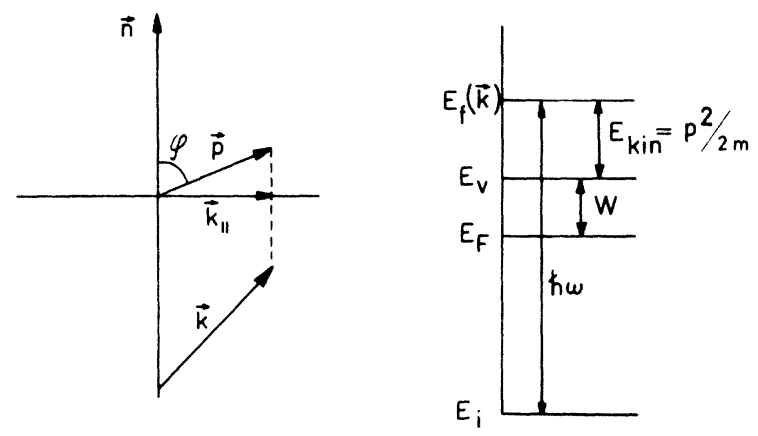

FIG. 13. Momentum of the final electron state inside $(\vec{k})$ and outside $(\vec{p})$ the crystal. The surface normal is indicated by $\vec{n}$. Right-hand part shows the energy diagram. $E_{v}$ is the vacuum level, $E_{F}$ the Fermi level, and $W$ the work function. The kinetic energy of the emitted electron is designated $E_{\mathbf{k} 1 \mathbf{n}}$. 

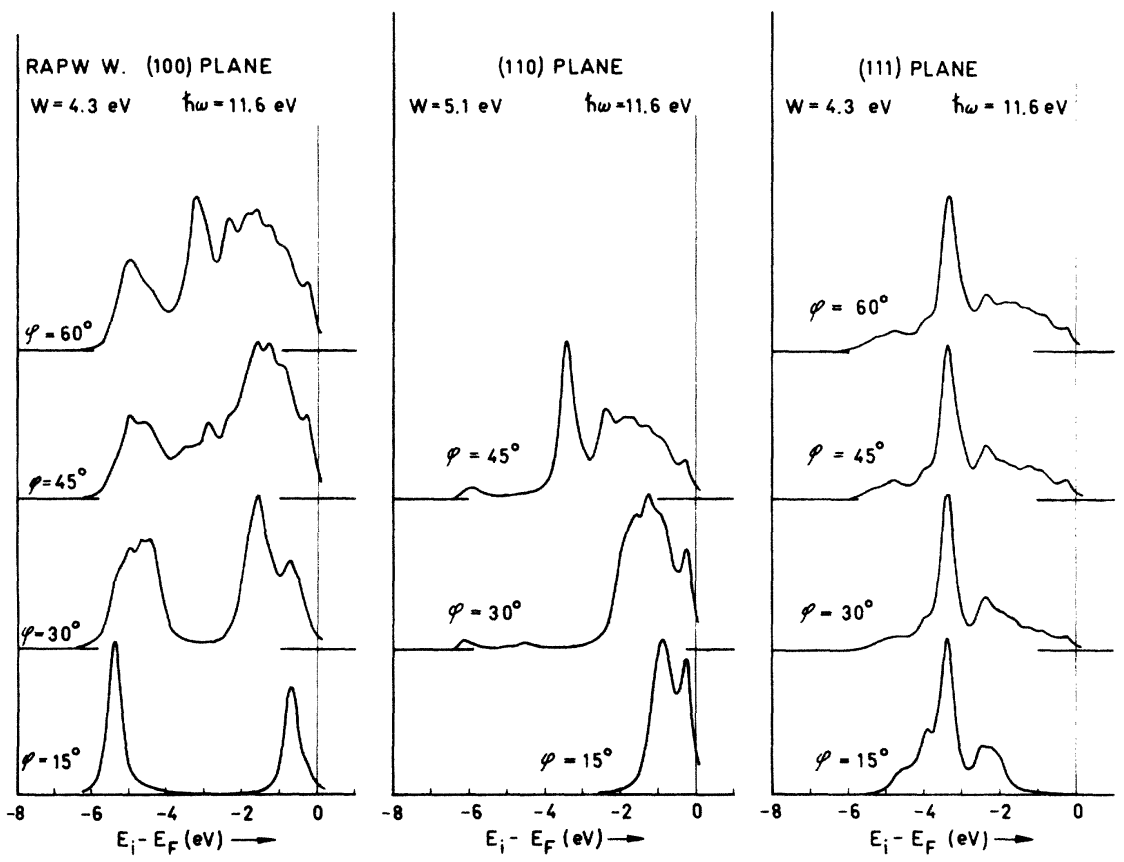

FIG. 14. Direct-model EDC's for $\hbar \omega=11.6 \mathrm{eV}$ corresponding to different acceptance angles $\phi$. All electrons emitted in a cone with half angle $\phi$ are included, $\phi$ being measured from $\vec{n}$ (Fig. 13).

is related to the one-dimensional energy distribution of the joint density of states along the symmetry line corresponding to the emitting surface plane. Since the experimental ${ }^{23}$ arrangement itself must half a finite acceptance cone angle $(\Delta \phi)$ this has been included in the present calculations. Figure 16 shows such direct EDC's for the (100) face. A half-angle $\Delta \phi$ of $6^{\circ}$ was used in order to account for a total acceptance angle of $12^{\circ}$ in the experiment described in Ref. 23. At low photon energies $(\hbar \omega<12 \mathrm{eV}$ ), only structure between -6 and -5 $\mathrm{eV}$ is predicted. The direct transitions that are responsible for this occur from the maximum of the lowest band (Fig. 2) halfway between $\Gamma$ and $H$ : For higher photon energies transitions from the higher bands are possible, and a strong peak between -2 and $-1 \mathrm{eV}$ appears. The spectral posi tion of this peak in the measured spectrum depends sensitively on the acceptance angle. This is demonstrated by the broken curve in Fig. 16 repre-
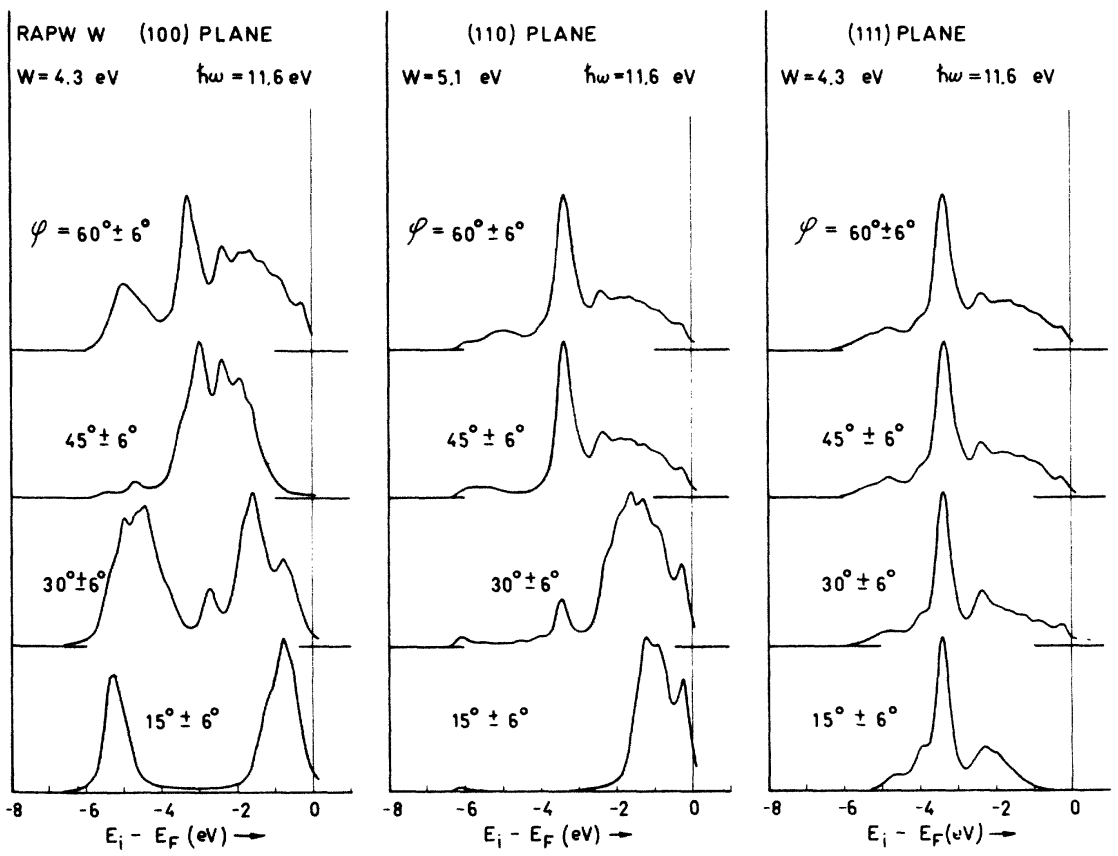

FIG. 15. Direct model EDC's for $\hbar$ ( $\omega=11.6 \mathrm{eV}$. Only those electrons that are emitted in direction $\phi \pm \Delta \phi$ are counted, where $\phi$ is a half-cone angle measured from the surface normal. 


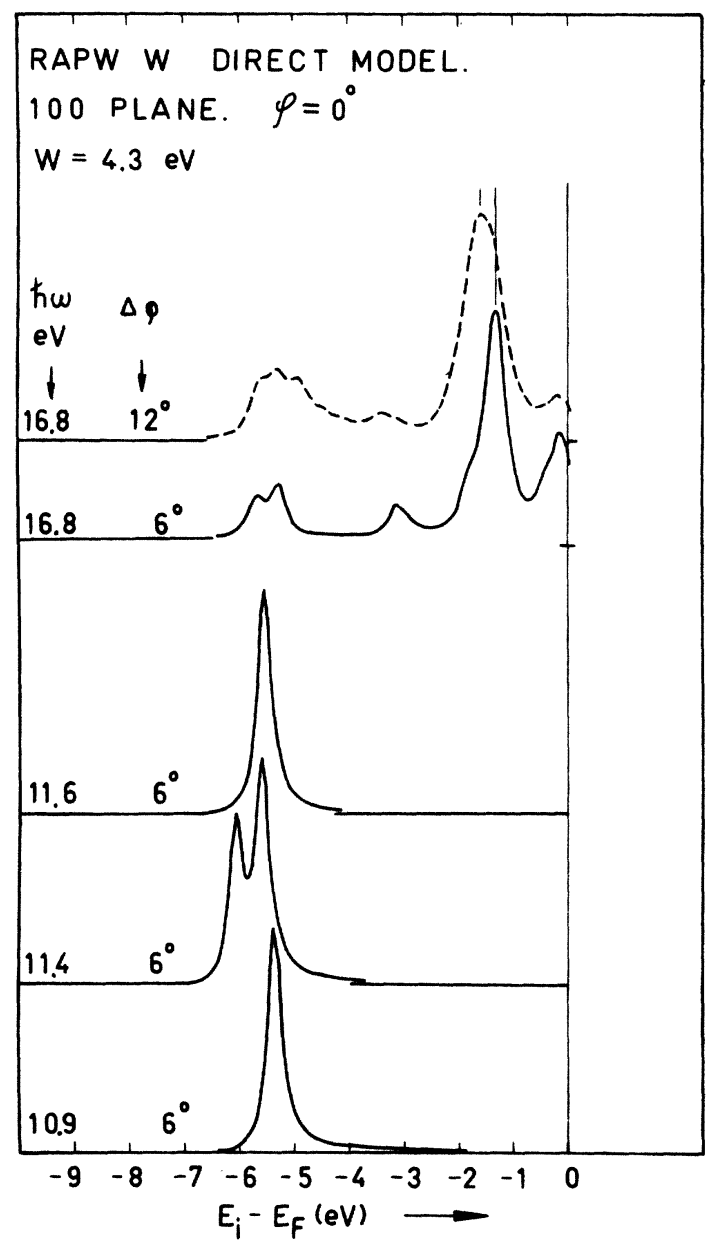

FIG. 16. Energy distribution curves calculated within the direct model for emission normal to a (100) face. A finite acceptance angle of $12^{\circ}$ has been assumed in all cases except for the broken curve which represents the $16.8 \mathrm{eV}$ spectrum with increased acceptance angle $\left(24^{\circ}\right)$.

senting the 16.8-eV EDC for $\Delta \phi=12^{\circ}$. The peak moves by $0.3 \mathrm{eV}$ if the acceptance angle is doubled.

The work function for the (110) face is ${ }^{23} 5.1 \mathrm{eV}$. This means that the vacuum level falls $0.8 \mathrm{eV}$ below the gap between band 6 and band 7 at $N$ (Fig. 2). Therefore, the direct model predicts that no photoemission should be observed in the [110] direction if the photon energy is between $9.4 \mathrm{eV}$ and $\hbar \omega_{\text {ons }}$ as defined earlier (Table VII). The onset energy is in the presently selected band structure $10.6 \mathrm{eV}$. For higher excitation energies, a peak is found right below $E_{F}$, as shown in Fig. 17. It corresponds to transitions from band 3 to band 7 near $N$. The extra peak appearing as a shoulder for $\hbar \omega \simeq 11 \mathrm{eV}$ is due to $3 \rightarrow 8$ transitions.

The energy-distribution curves for (111) emission have also been calculated within the direct model and are shown in Fig. 18 for photon ener- gies ranging from 10.2 to $13.0 \mathrm{eV}$. It is seen clearly how the previously mentioned strong - 3.5$\mathrm{eV}$ peak grows out of the spectrum for $\hbar \omega$ near $11.5 \mathrm{eV}$, whereas two distinct peaks dominate the EDC's for higher energies ( $\hbar \omega=12.2 \mathrm{eV})$. A more thorough discussion of these results is given in Ref. 23 where the calculations are compared to experimental spectra.

Figure 19 shows for comparison the nondirect model calculations of EDC's for normal emission from the (100), (110), and (111) faces. A nondirect spectrum for the experimental arrangement considered here essentially consists of the product of the density of states for energies below $E_{F}$ corresponding to the entire Brillouin zone and the onedimensional density of final states along the sym-

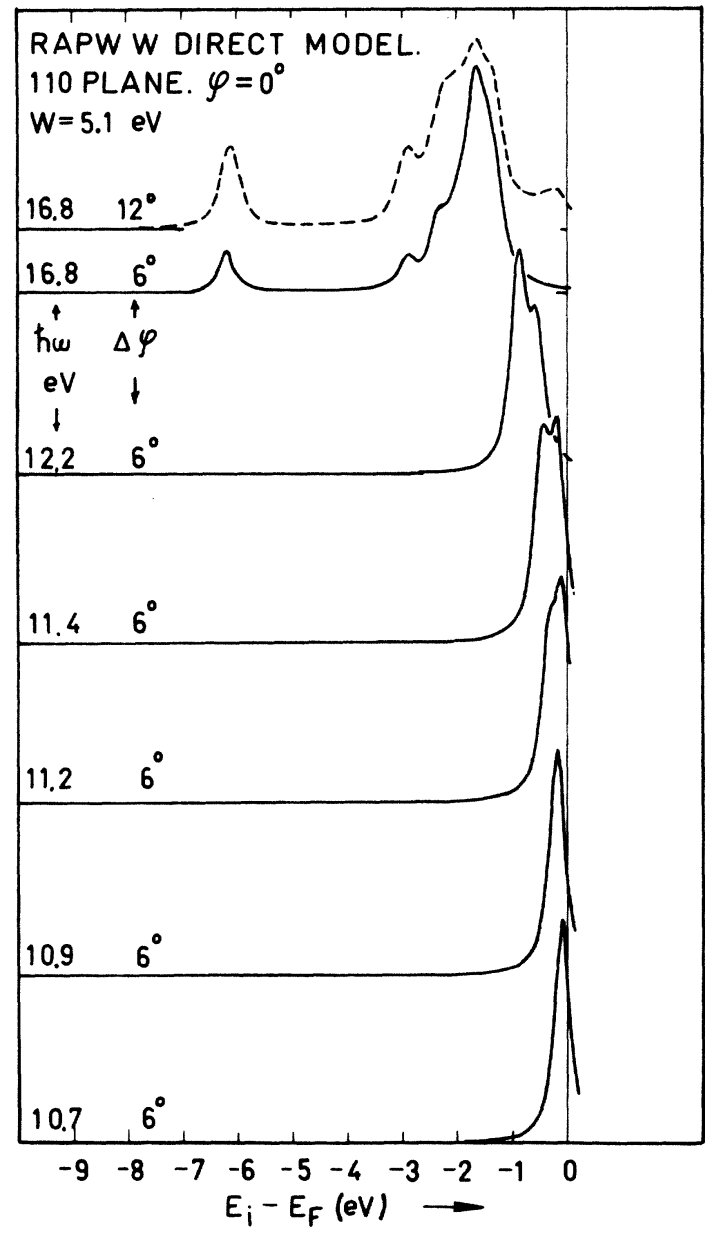

FIG. 17. Direct model EDC's for emission normal to a (110) face. The quantity $\Delta \phi$ is the half acceptance cone angle. The direct model predicts that no emission should be observed for photon energies between 9.4 and $10.6 \mathrm{eV}$. The lowest curve represents the calculated emission at the threshold energy $(10.7 \mathrm{eV})$ and corresponds to transitions from band 3 at the Fermi level to band 7 near the symmetry point $N$. 




FIG. 18. Direct model EDC's for the (111) face for photon energies between 10.2 and $13.0 \mathrm{eV}$.

metry line corresponding to the emission plane. No smooth cutoff functions have been applied, and only emission exactly normal to the surface is considered, i.e., $\Delta \phi=0$.

The one-dimensional density-of-states functions for each of the lines [100] $(\Delta),[110](\Sigma)$, and [111]
$(\Lambda)$ are presented in Figs. 20-22, where they are marked DOS. Little attention should be paid to the heights of the peaks since they all should be real infinities. The origin of the various peaks in the band structure may easily be established by comparing to Fig. 2. The numbers labeling each peak


FIG. 19. Non-direct distribution curves for emission normal to the three low-index faces. No smooth cutoff has been employed. Only emission in the normal direction $(\phi=0)$ is considered. The functions have been calculated as products of initial and final densities of states, where the density of initial states has been obtained by integration over the entire Brillouin zone and final density of states was taken one dimensional. 


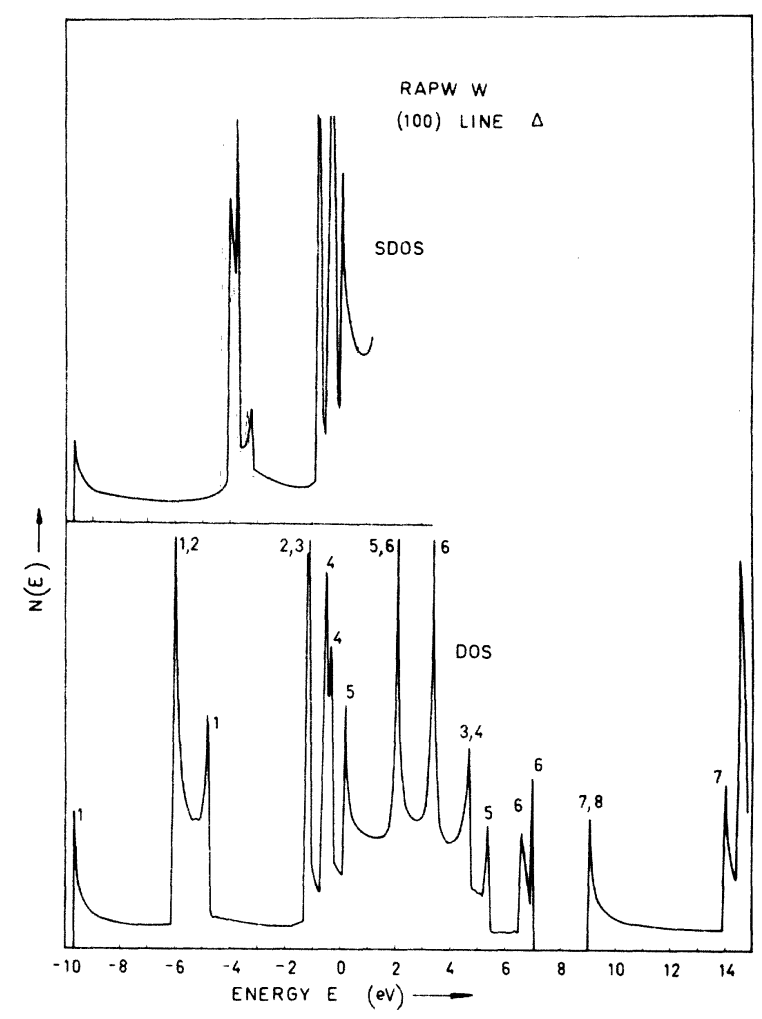

FIG. 20. One-dimensional density of states (DOS) along $\langle 100\rangle$ symmetry lines. The curve labelled SDOS is the surface density of states. The density-of-states functions in Figs. 20-22 are unbroadened, whereas those applied in Ref. 23 have been folded by a Lorentzian of $0.2 \mathrm{eV}$ full half-width.

refer to the band number that produces the peak. It is interesting to note that in all three DOS functions a clear gap is found in the final-state region. This gap extends from 7 to $9 \mathrm{eV}$ above the Fermi level at the $\Delta$ line, from 6 to 10.6 at $\Sigma$ and from 8.2 to $9.4 \mathrm{eV}$ at the $\Lambda$ line. Such gaps are expected to show up clearly in the part of the experimental photoemission spectra which is due to inelas tically scattered electrons ${ }^{72-74}$ Observation $^{23}$ of these gaps therefore provides (together with measurement of $\hbar \omega_{\text {ons }}$ ) a critical test of the band structure far above $E_{F}$.

\section{B. Surface contributions}

Experimental evidence ${ }^{23,24}$ shows that the observed photoelectric emission from tungsten cannot solely be attributed to bulk effects. This may be illustrated using the calculated data for the (110) face. The direct model predicts no photoemission normal to the (110) face for excitation energies between $9.4 \mathrm{eV}$ and the energy $\hbar \omega_{\text {ons }}=10.6$ $\mathrm{eV}$ which is the energy for transitions from the Fermi level to band 7 near the symmetry point $N$.
The same is predicted from the nondirect model as shown in the center panel of Fig. 19, except for a low-energy peak due to $6-\mathrm{eV}$ final state. This lack of photoemission within the bulk models is a cons equence of the large conduction-band gap along the $\Gamma N$ symmetry line (Fig. 2), since there are no final states available for transitions to occur, considering emission normal to the surface only. The experimental spectra, ${ }^{23}$ however, do not show a deficiency of normally emitted electrons in the above energy range as predicted by the bulk models. It is therefore concluded that another emission mechanism contributes to the observed spectra, and a likely explanation is that this is associated specifically with a surface effect.

The excitation and transport of excited electrons which was considered a three-step model for bulk emission is no longer relevant for excitation at the surface. Recent theories ${ }^{66,67}$ have treated photoelectric emission as a one-step process, and it has been shown ${ }^{75}$ that surface emission should reflect only the occupied part of the electronic level density at the surface, including surface states. Recent calculations ${ }^{76}$ predict the existence of surface states on the (100) face of tungsten in the $s-d$ gap. These have not been observed experimentally. Photoemission experi-

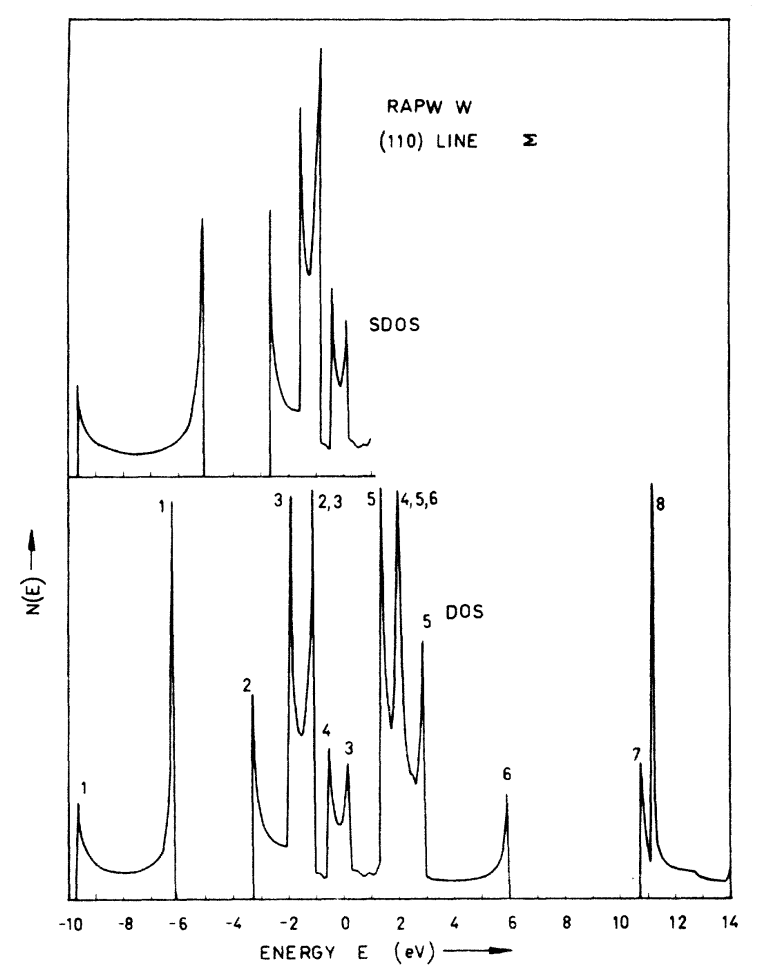

FIG. 21. One-dimensional density-of-states (DOS) and surface-density-of-states (SDOS) functions along the $\Sigma$ $\langle 110\rangle$ directions. 




FIG. 22. One-dimensional density-of-states (DOS) and surface-density-of-states (SDOS) functions along the $\Lambda$ $\langle 111\rangle$ directions.

ments ${ }^{24,77}$ do seem to indicate, however, the existence of a "surface resonance" at the (100) face associated with the spin-orbit gap close to the Fermi level, in agreement with field emission experiments. ${ }^{78}$ The present work does not include a calculation of such surface states. However, an attempt is made to derive a surface density of states, which is not identical to the bulk state density, based on a simple model, in order to allow a comparison to the surface contribution to photoelectric emission from tungsten.

Calculations of a surface density of states have been presented by Kalkstein and Soven ${ }^{79}$ and by Haydock and Kelly. ${ }^{80}$ It is not expected however, that a similar treatment is applicable to a material like tungsten so as to predict sufficient structure in the surface density of states as to allow a meaningful comparison to experimental spectra. The approach taken here is simply to deduce surface densities by a scaling method which accounts for the narrowing of the $d$ bands due to a reduced overlap between neighboring atoms. Scaling factors are deduced from the moments method $^{81}$ for the calculation of the density of states. The crude model applied here artificially conserves all structure in density-of-states function as the surface is approached from the bulk. The justification for such a simple calculation is given by subse- quent comparison and good agreement with experimental results. ${ }^{23}$

The second moment $\mu_{2}$ of a density -of-states function $N(E)$ essentially represents the square of the $\mathrm{rms}$ bandwidth. It is given by

$$
\mu_{2}=\int E^{2} N(E) d E,
$$

where the energy is measured from the band center. In the tight-binding model ${ }^{82}$ the second moment of the $d$ states is approximately ${ }^{81}$

$$
\mu_{2}=\sum_{i} n_{i}\left[d d \sigma^{2}(i)+2 d d \pi^{2}(i)+2 d d \delta^{2}(i)\right],
$$

where $n_{\boldsymbol{i}}$ is the number of neighbors of the $i$ th shell. The three two-center overlap integrals $d d \sigma, d d \pi$, and $d d \delta$ are defined in Ref. 82. In a bcc lattice only nearest and next-nearest neighbors contribute significantly to $\mu_{2}$, so the sum (9) only contains terms with $i=1$ and 2 . The rms width of the $d$ bands is then

$$
W_{d}(\mathrm{rms}) \propto\left(n_{1}+w_{2} n_{2}\right)^{1 / 2},
$$

with $n_{1}=8$ and $n_{2}=6$ for the bcc lattice. The weight factor $w_{2}$ is in the simplified tight-binding model above

$$
w_{2}=\frac{d d \sigma^{2}(2)+2 d d \pi^{2}(2)+2 d d \delta^{2}(2)}{d d \sigma^{2}(1)+2 d d \pi^{2}(1)+2 d d \delta^{2}(1)} .
$$

The overlap parameters have been deduced ${ }^{83}$ by fitting the tight-binding model (without spin-orbit coupling) to the nonrelativistic APW band structure calculated by Mattheiss ${ }^{5}$ (potential $V_{1}$ ), and using them in Eq. (11) we found $w_{2}=0.27$. An estimate by Haydock and Kelly ${ }^{80}$ suggests that the six nextnearest neighbors in the bcc lattice roughly are equivalent to 1.6 nearest neighbors, which also implies $w_{2}=0.27$. This exact agreement between the two results for $w_{2}$ is fortuitous; a calculation ${ }^{83}$ of $w_{2}$ from Eq. (11) for other bcc transition metals, $\mathrm{Mo}, \mathrm{Fe}, \mathrm{Cr}$, and other $\mathrm{W}$ band structures, gave values ranging from 0.18 to 0.30 .

The surface density of $d$ states is obtained by scaling the bulk $d$-band energies by $\left(n_{e s} / n_{e b}\right)^{1 / 2}$ relative to the "center of gravity." The "effective number of neighbors" is given by

$$
n_{e b, s}=n_{1 b, s}+w_{2} n_{2 b, s},
$$

the subscripts $b, s$ referring to "bulk" and "surface," respectively.

Another factor that might influence the surface density of states is the surface relaxation, i.e., effects due to a lattice spacing normal to the surface that is different from the lattice spacing in the bulk. Tentatively it has been suggested ${ }^{84}$ that the lattice is expanded by roughly $10 \%$ to the (100) and (110) surfaces, and $5 \%$ for the (111) surface. If this is true the effective $d-d$ overlap near the 
surface is further reduced. The relaxation effects could have been included in the present model if we had calculated $W_{d}$ for lattices deformed in the [100], and [111] directions. In view of the inaccuracies already inherent in our model an even simpler approach was taken. The relaxation narrowing was estimated from the hydrostatic deformation calculations (Fig. 4). The $d$-band narrowing factor, $f_{R}$, has been obtained from Fig. 4, using the entry $\Delta a / a$ equal to the relative change of the lattice constant due to relaxation multiplied by the relative number of nearest neighbors displaced. The estimated $d$ width $W_{d s}$ at the surface is then given by

$$
W_{d s}=W_{d} f_{R}\left(n_{e s} / n_{e b}\right)^{1 / 2} .
$$

The parameters $f_{R}, n_{1 s}$, and $n_{2 s}$ are summarized in Table VIII. The one-dimensional surface density-of-states functions as derived from the model described above are shown as the curves labeled SDOS in Figs. 20-22. The relaxation effect is most important for the (100) face. Realizing the uncertainty in $f_{R}$ we have therefore also shown (with a broken line in Fig. 20) a part of the SDOS function corresponding to $f_{R}=1$, i. e., a calculation excluding relaxation effects.

\section{SUMMARY AND CONCLUSIONS}

The electronic energy-band parameters for tungsten have been examined for eight different crystal potentials (Table I). Four of these potentials $V_{1}, V_{2}, V_{3}$, and $V_{4}$ were constructed in the same way, but refer to different lattice parameters. The temperatures corresponding to three of those lattices are approximately $T=4.2,300$, and 2150 $\mathrm{K}$. The remaining four potentials all refer to room temperature, but differ with respect to the exchange term and the electron configuration. Among the potentials $V_{2}, V_{5}, V_{6}, V_{7}$, and $V_{8}$ it was possible to select one, $V_{2}$, that leads to the best room-temperature band structure. This selection was made by comparing the calculated $\epsilon_{2}(\omega)$ profile to results of optical experiments covering photon energies up to $5 \mathrm{eV}$. The energy bands at the Fermi level obtained from $V_{1}$, which is the low-temperature analog to $V_{2}$ are shown to agree with the results of rf-size-effect measurements. In particular, the hole ellipsoids centered at the symmetry points $N$ have dimensions that depend critically on the potential. Low-temperature potentials constructed in the same manner as $V_{5}$, $V_{7}$, and $V_{8}$ lead to Fermi surfaces that disagree with experiments not only quantitatively but also qualitatively. The ad hoc muffin-tin potentials that in this way were shown to yield the best band structure of tungsten were obtained from relativistic Dirac-Slater atomic wave functions calculated
TABLE VIII. Parameters related to the simple model for obtaining the surface density of states. The number of nearest and next-nearest neighbors to a surface atom given by $n_{1 s}$ and $n_{2 s}$, and $f_{R}$ is the relaxation narrowing factor.

\begin{tabular}{clll}
\hline \hline & $(100)$ & $(110)$ & $(111)$ \\
\hline$n_{1 s}$ & 4 & 6 & 4 \\
$n_{2 s}$ & 5 & 4 & 3 \\
$f_{R}$ & 0.89 & 0.95 & 0.94 \\
$\left(\frac{n_{1 s}+w_{2} n_{2 s}}{n_{1 b}+w_{2} n_{2 b}}\right)^{1 / 2}$ & 0.745 & 0.857 & 0.707 \\
\hline \hline
\end{tabular}

for the ground state configuration $5 d^{4} 6 s^{2}$. Full Slater exchange was included in the atomic as well as in the muffin-tin potential.

The band structure of tungsten was calculated by means of the relativistic augmented plane wave method, and it covers the entire Brillouin zone. Density-of-states functions, joint density of states, and photoemission spectra were derived from this band structure. The $\epsilon_{2}(\omega)$ spectrum allows an interpretation of the optical reflectance measurements although the variation of the dipole matrix elements with $\vec{k}$ and energy has not been taken into account. A comparison of the density of states at the Fermi level to specific-heat measurements suggests an apparent electron-phonon enhancement of the thermal mass of $(10-20) \%$. However, in view of large experimental uncertainties this result is of little importance in a test of the band structure.

The strain response of the band structure has been obtained by comparing the calculations for four different lattice parameters $a$. The $d$-band width scales approximately as $a^{-5}$. The temperature coefficients for some particular energy gaps were estimated from the strain responses. The thermovariation of these gaps increases rapidly with temperature. At room temperature the temperature coefficient $d E_{g} / d T$ for the $1 \rightarrow 6$ transition at $N$ is estimated as $-8 \times 10^{-5} \mathrm{eV} / \mathrm{K}$, whereas its value at $T \simeq 1300 \mathrm{~K}$ is $-4.5 \times 10^{-4} \mathrm{eV} / \mathrm{K}$.

The present calculations serve as a basis for interpretation of directional photoemission experiments. For this purpose detailed calculations of the angular dependence of photoemission spectra have been performed. Energy distribution functions were derived from the band structure assuming conservation of the momentum component parallel to the surface during emission. Secondary cones have not been included in the calculated spectra. The EDC's for different acceptance angle but fixed photon energy obtained in this way depend sensitively on the cone angle. This has consequences not only for photoemission experiments 
on single crystals, but also if polycrystalline films $s^{85}$ are used since evaporated samples often exhibit a preferred orientation. Calculations of the EDC's were performed within the nondirect as well as the direct model. Only in one case, namely, for full angle emission from the (110) face with $\hbar \omega=16.8 \mathrm{eV}$ the calculated EDC's have been compared to experiment. In this particular case it seems that both bulk models agree equally well with the experiment with respect to the spectral position of the structure elements in the EDC. It follows, however, from the analysis of the directional EDC's in Ref. 23 that no evidence for nondirect transitions is found.

Calculations related to emission in directions normal to the (100), (110), and (111) faces have been studied in particular. The most significant result in this context is that neither the direct nor the nondirect model predicts any essential emission from the (110) face for $9.4 \mathrm{eV}<\hbar \omega<10.6 \mathrm{eV}$. This implies that emission observed in this direction and for photon energies below the onset at $10.6 \mathrm{eV}$ cannot be interpreted in terms of simple three-step processes in the bulk. It should, however, be emphasized that this conclusion is drawn from calculations that neglect secondary cones. It remains to be examined whether such higher order cones can contribute with sufficient intensity in the emission gap.

The calculated band structure exhibits gaps at the three symmetry lines $\Delta, \Sigma$, and $\Lambda$ at high energies. The (100) gap extends from 7 to $9 \mathrm{eV}$, the (110) gap from 6.0 to $10.6 \mathrm{eV}$, and the (111) gap is between 8.2 and $9.4 \mathrm{eV}$, all energies being measured from the Fermi level. These gaps are ex- pected to manifest themselves in that part of the photoemission spectra which is due to inelastically scattered electrons. Secondary electron emission spectra should also show these gaps. Such experiments thus provide tests of the band structure in the high-energy regime.

Parts of the photoemission spectra ${ }^{23}$ have been related to surface emission, which is considered as a one-step process where the electrons are excited from initial states near the surface directly into a vacuum state. These electrons carry information on the surface density of states (SDOS), and therefore estimates of such functions have been included here. The SDOS functions were calculated using a crude model that scales the $d$-band width by the square root of an effective number of neighbors to the atom considered. Further, crude estimates of relaxation effects were obtained from the strain responses. It is not clear how large the relaxation of the tungsten surfaces is, but tentatively we adopted the following values for expansion normal to the three faces, $10 \%$ for (100) and (110), and 5\% for the (111) face. This relaxation alone produces a narrowing of $d$-states which is approximately 11,5 , and $6 \%$ at the (100), (110), and (111) faces, respectively. In addition the $d$ bands at the surface are further narrowed due to the reduced number of neighbors. In the present model this narrowing alone amounts to 25,14 , and $30 \%$ for the (100), (110), and (111) faces.

\section{ACKNOWLEDGMENT}

The authors are grateful to Dr. R. F. Willis for his careful reading of the manuscript and valuable comments.
${ }^{1}$ J. H. Wood, Phys. Rev. 126, 517 (1962).

${ }^{2}$ L. F. Mattheiss, Phys. Rev. 134, A970 (1964).

${ }^{3}$ T. L. Loucks, Phys. Rev. Lett. 14, 693 (1965).

${ }^{4}$ T. L. Loucks, Phys. Rev. 139, A1181 (1965).

${ }^{5}$ L. F. Mattheiss, Phys. Rev. 139, 236 (1965).

${ }^{6}$ O. K. Andersen, Phys. Rev. B 2, 883 (1970).

${ }^{7}$ I. Petroff and C. R. Viswanathan, Phys. Rev. B $\underline{4}, 799$ (1970).

${ }^{8}$ N. E. Christensen, Phys. Status Solidi B 55, 117 (1973).

${ }^{9}$ N. E. Christensen, in Computational Solid State Physics, edited by F. Herman, N. W. Dalton, and T. Koehler (Plenum, New York, 1970), p. 155; Report No. 95, Physics Lab. I, Technical University of Denmark (unpublished).

${ }^{10}$ N. E. Christensen, Phys. Status Solidi 31, 635 (1969).

${ }^{11}$ N. E. Christensen, Phys. Status Solidi B 54, 551 (1972).

${ }^{12}$ N. E. Christensen and B. O. Seraphin, Phys. Rev. B 4, 3321 (1971).

${ }^{13}$ V. V. Boiko and V. A. Gasparov, Zh. Eksp. Teor. Fiz. 61, 2362 (1972) [Sov. Phys. -JETP 34, 1266 (1972)].

${ }^{14} \bar{E}$. Fawcett and D. Griffiths, J. Phys. Chem. Solids 23, 1631 (1962).

15 J. A. Rayne, Phys. Rev. 133, A1104 (1964).

${ }^{16}$ R. D. Girvan, A. V. Gold, and R. A. Phillips, J. Phys.
Chem. Solids 29, 1485 (1968).

${ }^{17}$ L. V. Nomerovannaya, M. M. Kirillova, and M. M. Noskov, Zh. Eksp. Teor. Fiz. 60, 748 (1971) [Sov. Phys. -JETP 33, 405 (1971)].

${ }^{18}$ N. E. Christensen, Phys. Status Solidi B 52, 241 (1972).

${ }^{19}$ N. E. Christensen, Phys. Lett. A 35, 206 (1971).

${ }^{20} \mathrm{I}$. Lindau and L. Walldén, Physica Scripta 3,77 (1971).

${ }^{21}$ N. V. Smith, Phys. Rev. B 3 , 1862 (1971).

${ }^{22}$ I. Lindau and L. Walldén, Solid State Commun. 9,209 (1971).

${ }^{23} \mathrm{~B}$. Feuerbacher and N. E. Christensen, following paper, Phys. Rev. B 10, 2373 (1974).

${ }^{24}$ B. Feuerbacher and B. Fitton, Phys. Rev. Lett. 29, 786 (1972).

${ }^{25}$ I. Adawi, Phys. Rev. 134, A788 (1964).

${ }^{26}$ J. G. Endriz, Phys. Rev. B 7, 3464 (1973).

${ }^{27}$ D. Grant, thesis (Pennsylvania State University, 1971) (unpublished).

${ }^{28}$ W. B. Pearson, A Handbook of Lattice Spacings and Structures of Metals (Pergamon, New York, 1958).

${ }^{29}$ F. C. Nix and D. MacNair, Phys. Rev. 61, 74 (1942).

${ }^{30}$ N. E. Christensen (unpublished).

${ }^{31} \mathrm{O}$. K. Andersen, thesis (Technical University of Denmark, 1969); and Ref. 6. 
${ }^{32}$ P. O. Löwdin, Adv. Phys. $\underline{5}, 1$ (1956).

${ }^{33}$ The eigenvalues are numbered from below in energy at each point in $\vec{k}$ space.

${ }^{34}$ W. M. Walsh and C. C. Grimes, Phys. Rev. Lett. 13 , 523 (1964).

${ }^{35}$ F. Herman and S. Skillman, Atomic Structure Calculations (Prentice-Hall, Englewood Cliffs, N. J., 1963).

${ }^{36}$ J. M. Ziman, Proc. Phys. Soc. Lond. 91, 701 (1967).

${ }^{37}$ G. Gilat and L. J. Raubenheimer, Phys. Rev. 144, 390 (1966).

${ }^{38}$ T. H. Geballe, Rev. Mod. Phys. 36, 134 (1964).

${ }^{39}$ American Institute of Physics Handbook, 2nd ed., edited by D. E. Gray et al. (McGraw-Hill, New York, 1963), pp. 4-61.

${ }^{40} \mathrm{~F}$. Heiniger, E. Bucher, and J. Muller, Physik Kondenseirten Materie 5, 243 (1966).

${ }^{41} \mathrm{E}$. Bucher, F. Heiniger, and J. Muller, Proceedings of the Ninth International Conference on Low Temperature Physics (Plenum, New York, 1965), p. 1059.

${ }^{42}$ W. L. McMillan, Phys. Rev. 167, 331 (1968).

${ }^{43}$ L. Hodges, R. E. Watson, and H. Ehrenreich, Phys. Rev. B 5, 3953 (1972).

${ }^{44} \mathrm{O}$. K. Andersen, Winter College on Electrons in Crystalline Solids 1972 (IAEA, Vienna, 1972).

${ }^{45}$ O. K. Andersen, Solid State Commun. 13, 133 (1973).

${ }^{46}$ V. Heine, Phys. Rev. 153, 673 (1967).

${ }^{44}$ R. L. Jacobs, J. Phys. C 1,492 (1968).

${ }^{48}$ W. J. Scouler, Phys. Rev. Lett. 18, 445 (1967).

${ }^{49}$ M. Cardona, Solid State Physics, edited by F. Seitz, D. Turnbull and H. Ehrenreich, (Academic, New York, 1969), Suppl. 11, p. 130.

${ }^{50}$ M. Garfinkel, J. J. Tieman, and W. E. Engeler, Phys. Rev. 148, 695 (1966).

${ }^{51}$ Note, however, that experimental difficulties may be encountered since a very high photon energy is required to observe the energy gaps discussed here.

${ }^{52}$ The band calculations discussed in Sec. II $D$ have not been extended to cover the entire Brillouin zone. The Fermi levels are determined by requiring it to be located in the SO gap at $\Delta$ (no lenses) and such that the jack dimensions are reasonable. If other criteria had been used to locate $E_{F}$, such as requiring reasonable hole ellipsoids to exist, the Fermi surface dimensions (and the qualitative features) would be in error at the $\Gamma H$ line.

${ }^{53}$ The conductivity $\sigma(\omega)$ is essentially $\epsilon_{2}(\omega) \omega$, i. e., in our model is $\sigma(\omega)$ proportional to $J(\hbar(\omega) / \omega$.

${ }^{54} \mathrm{C}$. N. Berglund and W. E. Spicer, Phys. Rev. 136 , A1030 (1964).

${ }^{55}$ C. N. Berglund and W. E. Spicer, Phys. Rev. $\underline{136}$, A1044 (1964).
${ }^{56}$ W. E. Spicer, Phys. Rev. 154, 385 (1967).

${ }^{57}$ P. -O. Nilsson, C. Norris, and L. Walldén, Solid State Commun. 7, 1705 (1969).

${ }^{58}$ P. -O. Nilsson, C. Norris, and L. Walldén, Physik Kondenseirten Materie 11, 220 (1970).

${ }^{59}$ D. E. Eastman and J. K. Cashion, Phys. Rev. Lett. 24, 310 (1970).

${ }^{60}$ N. V. Smith, Phys. Rev. B $\underline{4}, 1192$ (1972).

${ }^{61}$ N. V. Smith, Phys. Rev. Lett. 23, 1452 (1969).

${ }^{62}$ N. V. Smith and M. M. Traum, Phys. Rev. Lett. 25, 1017 (1970).

${ }^{63}$ A. R. Williams, J. F. Janak, and V. L. Moruzzi, Phys. Rev. Lett. 28, 671 (1972); Phys. Rev. B 8, 2546 (1973).

${ }^{64}$ The same threshold function as applied to Rh (Ref. 8) has been used here.

${ }^{65}$ J. M. Baker and D. E. Eastman, J. Vac. Sci. Technol. 10,223 (1973).

${ }^{66}$ G. D. Mahan, Phys. Rev. B 2,4334 (1970).

${ }^{67}$ W. L. Schaich and N. W. Asheroft, Solid State Commun. 8,1959 (1970).

${ }^{68}$ T. Gustafson, P. -O. Nilsson, and L. Walldén, Phys. Lett. A 37, 121 (1971).

${ }^{69}$ E. O. Kane, Phys. Rev. Lett. 12, 97 (1964).

${ }^{70}$ Results not shown in the figures may be obtained from the authors.

${ }^{71} \mathrm{As}$ a matter of convenience are all spectra scaled to the same maximum amplitude.

${ }^{72}$ E. O. Kane, Phys. Rev. 159, 624 (1967).

${ }^{73} \mathrm{P}$. -O. Nilsson and I. Lindau, in Band Structure Spectroscopy of Metals and Alloys, edited by D. J. Fabian and L. M. Watson (Academic, New York, 1973), p. 55. ${ }^{74}$ H. Kanter, Phys. Rev. B 1, 522 (1970).

${ }^{75}$ W. L. Schaich and N. W. Ashroft, Phys. Rev. B $\underline{3}$, 2452 (1971).

${ }^{76}$ S. J. Gurman and J. B. Pendry, Phys. Rev. Lett. 31 , 637 (1973).

${ }^{77}$ B. J. Waclawski and E. W. Plummer, Phys. Rev. Lett. 29, 783 (1972).

${ }^{78}$ E. W. Plummer and A. E. Bell, J. Vac. Sci. Technol. 9, 583 (1972).

${ }^{79}$ D. Kalkstein and P. Soven, Surf. Sci. 26, 85 (1971).

${ }^{80}$ R. Haydock and M. J. Kelly, Surf. Sci. 38,139 (1973).

${ }^{81} \mathrm{~F}$. Ducastelle and F. Cyrot-Lackmann, J. Phys. Chem. Solids 31,1295 (1970).

${ }^{82}$ J. C. Slater and G. Koster, Phys. Rev. 94, 1498 (1954).

${ }^{83}$ N. E. Christensen (unpublished).

${ }^{84}$ R. F. Willis (private communication).

${ }^{85}$ R. Y. Koyama and R. L. Hughey, Phys. Rev. Lett. 29, 1518 (1972). 